J.-M. Lourier, M. Stöhr, B. Noll, S. Werner and A. Fiolitakis, Scale Adaptive Simulation of a thermoacoustic instability in a partially premixed lean swirl combustor, Combustion and Flame 183 (2017) 343-357.

The original publication is available at www.elsevier.com

http://dx.doi.org/10.1016/j.combustflame.2017.02.024 


\title{
Scale Adaptive Simulation of a thermoacoustic instability in a partially premixed lean swirl combustor
}

\author{
J.-M. Lourier ${ }^{\mathrm{a}}$, M. Stöhr*, B. Noll, S. Werner, A. Fiolitakis \\ Institute of Combustion Technology, German Aerospace Center (DLR), Pfaffenwaldring 38-40, 70569 Stuttgart, Germany \\ ${ }^{a}$ Present address: GE Aviation, Freisinger Landstr. 50, 85748 Garching near Munich, Germany
}

\begin{abstract}
The thermoacoustic oscillation of a turbulent, swirl-stabilized, partially premixed flame in the PRECCINSTA gas turbine model combustor is analyzed by means of a Scale Adaptive Simulation (SAS) method. In the critical regions of the combustor the SAS features a fine spatial resolution and thus corresponds to a Large Eddy Simulation (LES). Two combustion models are applied, a simple eddy dissipation model and a detailed finite rate chemistry (FRC) model. A previous LES for the same combustor by Franzelli et al. [Combust. Flame 159 (2012) 621-637] indicated that the acoustic impedance of the fuel supply plays a critical role. Therefore in the present work, the fuel channels and fuel plenum are included in the computational domain and thereby the fuel inlet impedance is inherently taken into account. The resulting fields of velocity, temperature and mixture fraction fit well to experimental data with a slightly better agreement for the detailed FRC model. For both combustion models the computed frequency of the thermoacoustic oscillation is close to the experimental value, whereas its amplitude is significantly overestimated by about $15 \mathrm{~dB}$ in comparison to measurements. The reason for this overestimation is analyzed using an additional measurement where acoustic damping due to vibrating side walls is suppressed. For the latter experiment both frequency and amplitude agree well with CFD results, which indicates that acoustic damping effects must be carefully taken into account for validation of CFD. The 3D time-resolved simulations further provide detailed insights into the interaction of flow and mixing in the swirler, which leads to a convective time-lag between oscillations of velocity and equivalence ratio in the flow of unburned gas that largely affects the heat release response of the flame. Taken together, the results show that SAS computations can accurately reproduce frequency and amplitude of thermoacoustic oscillations of turbulent partially premixed flames in a gas turbine combustor provided that proper modeling of fuel supply and acoustic boundary conditions is applied.
\end{abstract}

Keywords: Scale Adaptive Simulation, Thermoacoustic instability, Partially-premixed combustion, Acoustic damping, Gas turbine combustion

\section{Introduction}

Modern gas turbines employ lean premixed combustion since this results in a lower flame temperature, and hence in less thermal nitrogen oxide [1]. However, lean premixed combustion increases the susceptibility to thermoacoustic instabilities, which occur if the combustion dynamics couples with an acoustic eigenmode of the combustor [2]. Since these instabilities can cause severe operational difficulties, their prediction is of great importance in the design process of combustion chambers. Within the last decade Large Eddy Simulation (LES) has been established as a tool that can support this design process as it resolves the energy containing turbulent scales of a flow, which often increases significantly the accuracy of a turbulent flame calculation in combustors with complex geometry [3,4]. LES of thermoacoustic instabilities, in particular, is especially sensitive to acoustic boundary conditions $[5,6,7]$ and to the dynamics of fuel-air mixing $[8,9]$ and turbulence-chemistry interaction [10]. The application of appropriate boundary conditions

\footnotetext{
${ }^{*}$ Corresponding author.

Email address: michael.stoehr@dlr.de (M. Stöhr)
}

and model closures for flow, mixing and reaction is therefore a most critical, yet generally unresolved issue for an accurate and at the same time computationally affordable modeling of these instabilities.

The present work describes a detailed numerical simulation of a thermoacoustic instability focusing on the proper choice and evaluation of acoustic boundary conditions and model closures. The simulation is applied to a turbulent swirl-stabilized flame in a partially premixed swirl combustor that is typical for gas turbines, namely the so-called PRECCINSTA combustor [11]. For the chosen operating condition, detailed measurements of velocity field and thermochemical states by Meier et al. [12] are used for validation.

Compared to fully premixed operation, thermoacoustic oscillations in partially premixed combustors exhibit an additional complexity as they are driven not only by fluctuations of velocity but also by fluctuations of equivalence ratio in the unburned gas entering the flame zone $[13,14,8,15,16]$. Therefore, the mixing of fuel and air by the turbulent flow in the premixing zone of the combustor has to be well resolved in order to predict the thermoacoustic instability properly. In this 
work turbulent fluctuations are modeled using the Scale Adaptive Simulation (SAS) technique, which allows wide variations of the grid resolution within the computational domain. In regions with appropriately high grid resolution, an LES solution is obtained that resolves most of the energy containing turbulent scales of the reacting flow. In case of low grid resolution, an Unsteady Reynolds Averaged Navier-Stokes (URANS) solution is obtained. A grid resolution that is sufficiently high for an LES solution is chosen in the combusting and premixing regions of the flow.

The PRECCINSTA combustor has been analyzed numerically in several previous studies [11, 17, 18, 19, 20, 6, 21, 22]. Most of these works [11, 17, 18, 19, 20, 22], however, investigated either stable operating conditions or assumed a perfectly premixed flame. For the same combustor and operating condition as in this work, the frequency of the thermoacoustic oscillation was computed in an LES by Franzelli et al. with a discrepancy of $100 \mathrm{~Hz}$ to the measured data [6]. According to the authors, this deviation might result from inaccurate modeling of the acoustic impedance at the fuel inlet boundaries in the swirler. Therefore in the present work the fuel injection holes and the fuel plenum are included in the computational domain and hence the fuel inlet impedance is inherently taken into account.

An accurate prediction of a thermoacoustic instability also requires precise modeling of the combustor acoustics, which depends strongly on the acoustic boundary conditions [23, 24, 25, 6, 7]. Time Domain Impedance Boundary Conditions (TDIBC) allow to apply acoustic filters of arbitrary order at domain boundaries, and hence to precisely control acoustic wave reflections [26, 27, 28]. TDIBC are therefore used in the present work to model the acoustic outlet boundary condition. The computations are carried out with two combustion models, namely a simple yet computationally efficient Eddy Dissipation Model (EDM) and a detailed direct finite-rate chemistry model (FRC). The results from both models are evaluated by a comparison to detailed experimental data including velocity, mixture fraction, temperature and acoustic spectra, and to the previous LES by Franzelli et al.

While the oscillation frequencies of the present SAS computations agree well with the measured values, the corresponding amplitudes of the oscillation are markedly higher than in the experiment. It is suspected that this difference is caused by acoustic damping due to loosely mounted quartz glass windows that were used as combustion chamber side walls in the experiments with optical diagnostics [12]. To test this hypothesis, additional acoustic measurements are performed where the combustor is equipped with rigid metal walls, and compared to the numerical results. Finally, the CFD results are used to examine the mechanism of fuel-air mixing in the swirler nozzle, which leads to the formation of equivalence ratio oscillations and thus significantly affects the dynamics of the thermoacoustic instability.

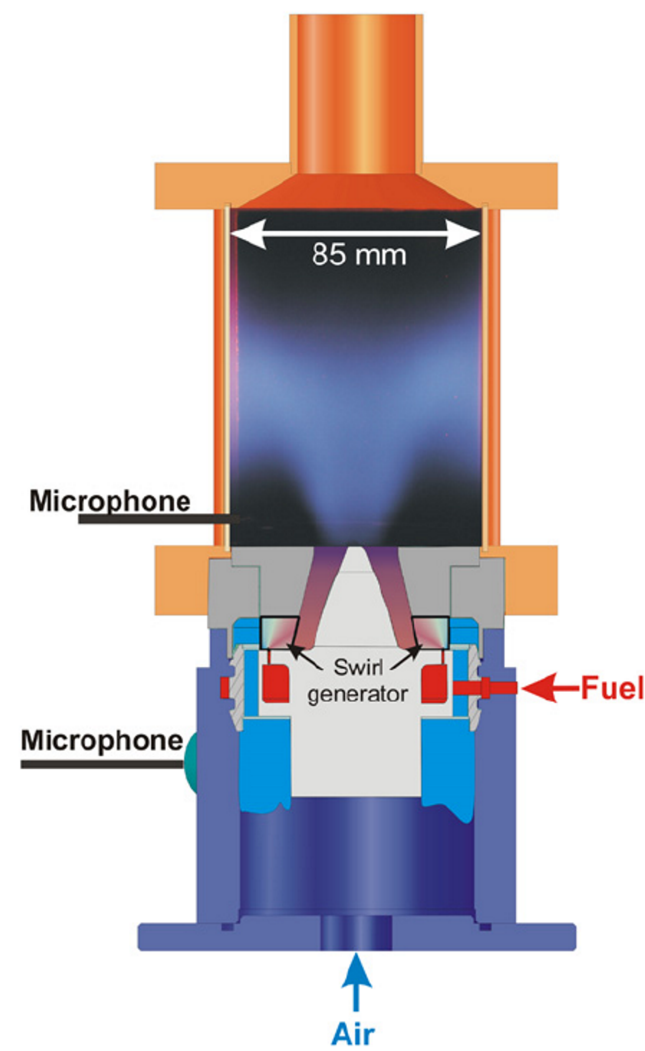

Figure 1: Sketch of the PRECCINSTA combustor.

\begin{tabular}{ll}
\multicolumn{2}{c}{ Table 1: Operating condition } \\
Thermal power & $\mathrm{P}_{\mathrm{th}}=25.1 \mathrm{~kW}$ \\
\hline Equivalence ratio & $\phi=0.70$ \\
\hline Air mass flow & $\dot{m}_{\text {air }}=734.2 \mathrm{~g} / \mathrm{min}$ \\
\hline Fuel mass flow & $\dot{m}_{\mathrm{CH} 4}=30 \mathrm{~g} / \mathrm{min}$ \\
\hline Inlet temperature & $T=320 \mathrm{~K}$ \\
\hline Pressure & atmospheric
\end{tabular}

\section{Experimental setup}

\subsection{Gas turbine model combustor}

The combustor shown in Fig. 1 was designed within the EU project PRECCINSTA [29, 11] and is derived from an industrial gas turbine by Turbomeca (now Safran Helicopter Engines). Air first enters a cylindrical plenum $(d=78 \mathrm{~mm})$ and then passes through a swirl generator with 12 radial vanes and a converging nozzle $(d=27.85 \mathrm{~mm})$ with a central conical bluff body into the combustion chamber. The chamber has a square cross section of $85 \times 85 \mathrm{~mm}$ and a height of $114 \mathrm{~mm}$, and the exit is cone shaped leading to a short central exhaust pipe ( $d=40$ $\mathrm{mm}$ ). The combustor is operated at atmospheric pressure and in partially premixed mode, i.e., the gaseous fuel $\mathrm{CH}_{4}$ is injected through 12 holes into the swirler vanes as shown in Fig. 1. 


\subsection{Operating condition and validation data}

For the present combustor, flames at different operating conditions have been characterized experimentally by the Institute of Propulsion Technology and the Institute of Combustion Technology of the German Aerospace Center (DLR) $[12,11]$. The three-dimensional velocity field was measured by laser Doppler velocimetry (LDV), and the flame structure by $\mathrm{OH}^{*}$ chemiluminescence and planar laser induced fluorescence (PLIF) of $\mathrm{OH}$. Major species concentrations, mixture fraction and temperature were obtained by laser Raman scattering. The present work focuses on the operating condition detailed in Table 1 with an equivalence ratio of $\phi=0.7$ and a thermal power of $P_{\mathrm{th}}=25 \mathrm{~kW}$ where a pronounced thermoacoustic instability with a frequency of about $280 \mathrm{~Hz}$ occurs.

Pressure measurements were carried out as part of this work using acoustic sensors at two locations, one in the air plenum and one in the combustion chamber as shown in Fig. 1. Depending on the pressure amplitude, either microphones (Brüel \& Kjær type 4939) or piezo-resistive sensors (Kistler 4043A) are used. In the plenum the sensor is flush mounted to the wall, whereas for the chamber an acoustic probe is used where the sensor is located $\approx 15 \mathrm{~cm}$ away from the chamber in order to protect it from heat. The acoustic effects of the probe are determined by a calibration, and the measured data is corrected for these effects [30].

In the experimental work of Meier et al. [12], the combustion chamber side walls were made of quartz glass plates, which are loosely mounted in the corner posts of the chamber in order to avoid thermal stress. Due to the loose support, the side walls are vibrating and not fully gas-tight, which may lead to damping of the thermoacoustic oscillation. In order to assess the influence of the quartz windows and their support on the thermoacoustic oscillation, additional pressure spectra were measured within this work, both for the original configuration with quartz glass windows and a modified configuration with rigid metal walls. The metal walls are mounted tightly in the corner posts such that the damping at the combustion chamber side walls is largely suppressed. For both configurations a choked orifice plate is installed in the air supply $15 \mathrm{~cm}$ upstream of the plenum in order to provide a well-defined acoustic inlet boundary. It is noted that for the present measurements with quartz windows the thermoacoustic frequency is around $15 \mathrm{~Hz}$ lower compared to the previous experiments of Meier et al. [12] where no sonic orifice was present.

\section{Scale Adaptive Simulations}

\subsection{THETA code and numerical mesh}

In this work the Favre filtered transport equations for mass, momentum, enthalpy and species mass fractions [31] are solved by means of the DLR CFD software THETA (Turbulent Heat Release Extension of the TAU code). THETA is a second order accurate, unstructured, finite volume code optimized for low Mach number combustion [32]. Transport equations are solved on a dual-grid of polyhedral cells using efficient matrix-free linear or multi-grid solvers [32]. The pressurevelocity coupling methods SIMPLE [33], projection [34] and SICS (Semi-Implicit Characteristic Splitting) [35, 36] are implemented. SICS enables the computation of (compressible) acoustic waves and is highly efficient for low Mach number flows. It was specifically developed to resolve acoustic oscillations in gas turbine combustion chambers [37] and is thus applied in the present simulation. Furthermore, various models for turbulence (URANS, LES, hybrid LES/RANS), combustion [38, 39, 40, 41] and soot formation [42, 43, 44] are available. Additionally, THETA can be coupled with a Lagrangian multiphase method [45].

In the present work, the computational domain is discretized by approximately $44 \mathrm{M}$ tetrahedral cells and prism layers at swirler and combustion chamber walls, which corresponds to a numerical grid of around $8 \mathrm{M}$ points. The grid spacing $\Delta x$ varies widely over the computational domain as shown in Fig. 2a. The highest spatial resolution of $\Delta x \approx 0.04 \mathrm{~mm}$ is implemented in the region near the fuel injection channels, and the lowest resolution of $\Delta x \approx 3 \mathrm{~mm}$ is present in the air plenum. In the flame region the resolution is approximately $0.75 \mathrm{~mm}$.

The dissipation and dispersion errors of acoustic wave propagation are highest in the plenum due to the large grid spacing and low speed of sound of about $340 \mathrm{~m} / \mathrm{s}$. For frequencies up to $2 \mathrm{kHz}$, this corresponds to a resolution of more than 50 points per wave length (PPW), which results in maximum dissipation and dispersion errors of less than $0.5 \%$ and $0.05 \%$, respectively $[46,47]$. Furthermore, a time step of $\Delta t=0.5 \mu \mathrm{s}$ is used for the simulations. This leads to a temporal resolution of acoustic waves with at least 1000 points per period (PPP) up to a frequency of $2 \mathrm{kHz}$. A pure central difference scheme is used for the convection term of the momentum equation. The CFL number $C=u \Delta t / \Delta x$ based on the flow velocity $u$ is $C<0.2$ in the combustion chamber and plenum and $C<1$ in the burner nozzle, swirler and fuel injection channels. Therefore, the discretization of the combustor ensures accurate prediction of acoustic wave propagation and turbulent velocity fluctuations within the whole computational domain.

\subsection{Modeling of turbulent flow and transport}

The turbulent flow is computed using the Scale Adaptive Simulation (SAS) approach, where a certain part of the unsteady flow is resolved explicitly and the other part is modelled by a RANS solver using the $k-\omega$ SST model by F. Menter [48]. The latter combines the standard $k-\epsilon$ model, which generally performs better in regions remote from walls, and the standard $k-\omega$ model that provides more accurate solutions near walls. The transition between the $k-\epsilon$ and the $k-\omega$ model is controlled by appropriate blending functions specified in Ref. [48]. While for the resolved part of the flow the scalar transport equations are directly solved, the subgrid scale SGS scalar fluxes are modeled by a classical gradient diffusion assumption [49] where turbulent Prandtl and Schmidt numbers are set to 0.7.

The SAS model, which is described in detail in Ref. [50], controls the transition between modelled to the resolved part by 


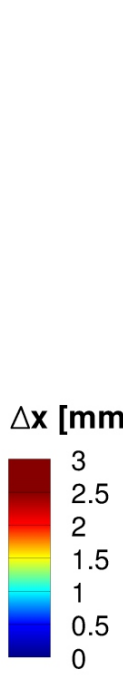

a
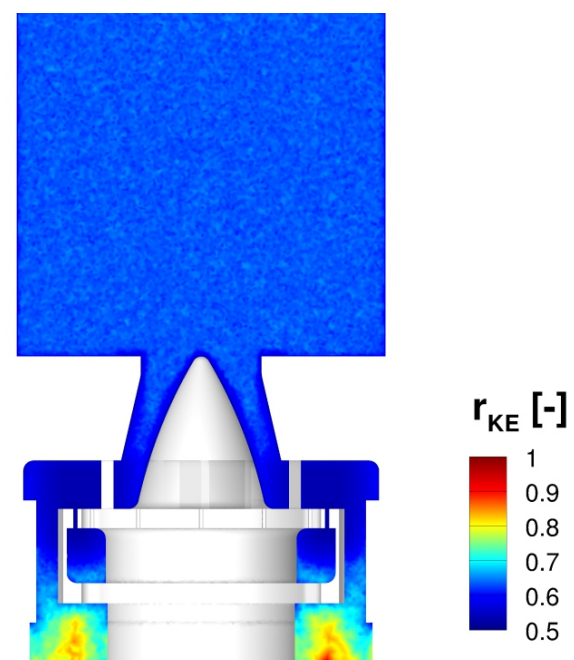

b

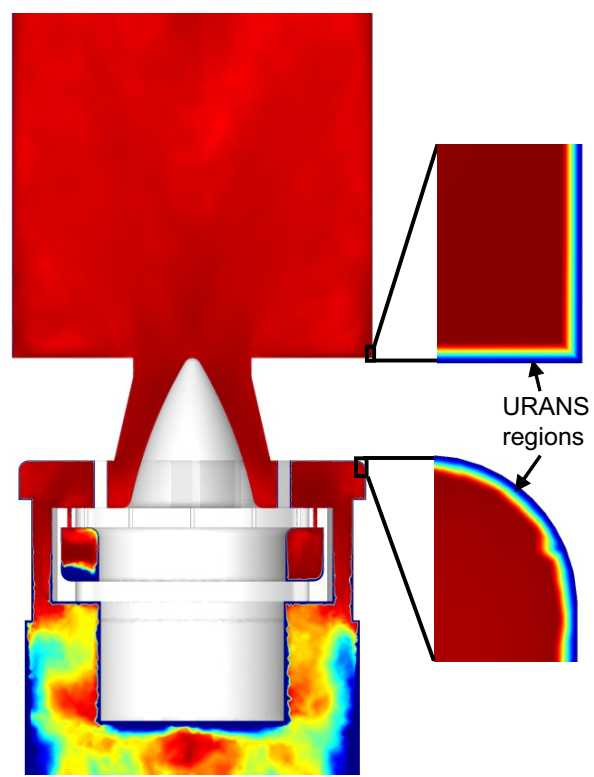

Figure 2: Numerical grid and ratio of resolved to total turbulent kinetic energy.

adding the forcing term

$$
\begin{aligned}
& F_{\mathrm{SST}-\mathrm{SAS}}= \\
& \max \left[C_{F 1} k S^{2} \frac{L_{t}}{L_{\mathrm{VK}}}-\frac{2}{C_{F 2}} k \max \left(\frac{1}{\omega^{2}} \frac{\partial \omega}{\partial x_{j}} \frac{\partial \omega}{\partial x_{j}}, \frac{1}{k^{2}} \frac{\partial k}{\partial x_{j}} \frac{\partial k}{\partial x_{j}}\right), 0\right]
\end{aligned}
$$

to the $\omega$ equation, where $L_{t}$ is the turbulent length scale, $L_{\mathrm{vK}}$ is the von Karman length scale and $C_{F 1}$ and $C_{F 2}$ are model constants [48]. The forcing term $F_{\text {SST-SAS leads to a conversion of }}$ energy from the modelled to the resolved part depending on the von Karman length scale. In regions of low grid resolution, the value of $L_{\mathrm{VK}}$ is high and the resulting forcing is low, and therefore the solution corresponds to an URANS where only a small part of the flow is resolved. In regions of high grid resolution and unsteady flow, the value of $L_{\mathrm{vK}}$ decreases. This leads to an increased forcing such that the turbulent viscosity decreases, and hence the fraction of modelled turbulent fluctuations is reduced and the resolved part of the unsteady flow rises. When the latter is sufficiently high as discussed in the paragraph below, the solution is referred to as an LES. It is noted that the usage of SAS in conjunction with the SST $k-\omega$ model has the major advantage of obviating the requirement of high grid resolution near walls that is typical for common pure LES models.

The resulting smooth transition between an URANS and an LES solution is assessed by the ratio between resolved and total turbulent kinetic energy

$$
r_{\mathrm{KE}}=\frac{\overline{u_{f}^{2}}}{\overline{u_{f}^{2}}+2 \bar{k}}
$$

where $u_{f}$ and $k$ are the resolved velocity fluctuations and the modeled turbulent kinetic energy, respectively, and the overbar denotes temporal averaging. According to Davidson [51] the ratio $r_{\mathrm{KE}}$ should exceed $90 \%$ in order to achieve a proper LES solution. Figure $2 \mathrm{~b}$ shows this ratio for the numerical grid used for the present computations. Within the swirler and the combustion chamber, the ratio of the turbulent kinetic energies is above $90 \%$, and hence according to the criterion for $r_{\mathrm{KE}}$ the grid is adequate for a proper LES modeling of turbulence. By contrast this criterion is not met within the plenum, and hence an URANS solution is obtained in these regions of the computational domain.

Within the first cell layers at the wall an additional wall function for the turbulent $k$ - $\omega$ equations of the SAS model is applied. This ensures that the resolved share of turbulent fluctuations $r_{\mathrm{KE}}$ approaches zero, i.e., the wall boundary layer is treated as URANS. As can be seen in the zoomed parts of Fig. 2b, the URANS regions at the walls (marked blue) are limited to the first prism layers of the grid. For the combustion chamber walls, the $y^{+}$values of the first grid node off the wall are $y^{+}<1$, which complies with the underlying $k-\omega$ model used in the URANS regions.

\subsection{Combustion modeling}

The simulation is performed with two different combustion models in order to assess the influence of the combustion model on the predicted thermoacoustic instability. Firstly, the EddyDissipation model (EDM) [41, 52, 49], which is widely used for industrial applications due to its computational efficiency [53], is applied in conjunction with a two-step reaction mechanism. Secondly, a direct detailed finite-rate chemistry model (FRC) is used, which incorporates a detailed reaction scheme and an assumed probability density function (PDF) method [32] for subgrid modeling. Both combustion models are presented in more detail in the following.

The physical runtime of the simulations was $90 \mathrm{~ms}$ for EDM, whereas for FRC it was limited to $75 \mathrm{~ms}$ due to the high compu- 

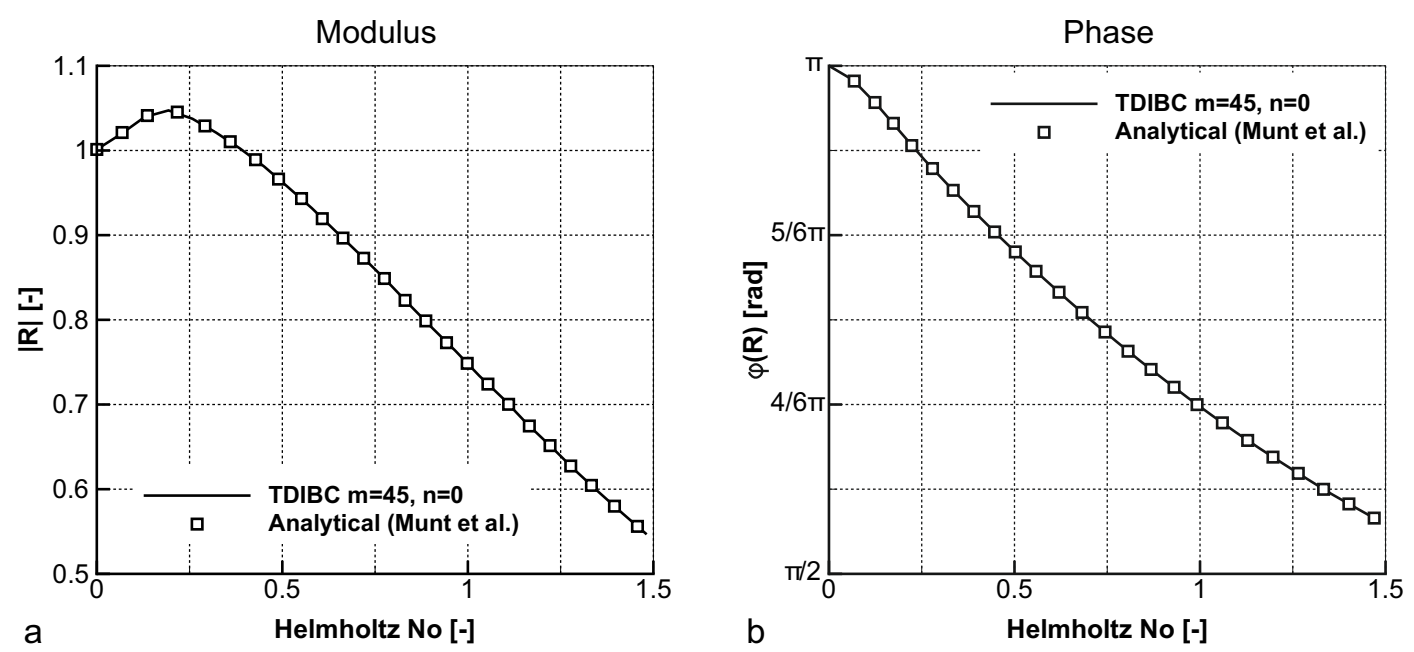

Figure 3: (a) Modulus and (b) phase of the reflection coefficient at the atmospheric outlet of the combustor.

tational costs. The corresponding computational runtimes were 8.3 days on 128 CPU cores for EDM and 16.5 days on 256 cores for FRC.

\subsubsection{Eddy Dissipation Model}

In case of the EDM approach reaction rates are modeled by the two-step methane mechanism BFER proposed by Franzelli et al. [6], which takes into account the methane oxidation and the carbon-oxide equilibrium according to

$$
\begin{aligned}
\mathrm{CH}_{4}+\frac{3}{2} \mathrm{O}_{2} & \rightarrow \mathrm{CO}+2 \mathrm{H}_{2} \mathrm{O} \\
\mathrm{CO}+\frac{1}{2} \mathrm{O}_{2} & \rightleftharpoons \mathrm{CO}_{2} .
\end{aligned}
$$

On the one hand, the chemical reaction rates are computed under the assumption of fast chemistry by

$$
R R_{r}^{\mathrm{EDM}}=A \rho \frac{1}{\tau_{t}} \min \left(\frac{Y_{e}}{v_{e, r}^{\prime} M_{e}}, B \frac{\sum_{p} Y_{p}}{\sum_{p} v_{p, r}^{\prime \prime} M_{p}}\right),
$$

where $R R_{r}$ is the reaction rate of reaction $r, \tau_{t}$ is the turbulent time scale, $Y_{e}$ and $Y_{p}$ are the filtered mass fractions of a reactant and a product, respectively, $v_{e, r}^{\prime}$ the forward stoichiometric coefficient, $v_{p, r}^{\prime \prime}$ the backward stoichiometric coefficient, $M_{e}$ the molar mass of a reactant, $M_{p}$ the molar mass of a product, $\rho$ the filtered density and $A=4$ and $B=0.5$ are model constants. The turbulent time scale is computed from the turbulence frequency $\omega$ provided by the SST $k-\omega$ model described in Sect. 3.2 as $\tau_{t}=1 / \beta_{k} \omega$ with $\beta_{k}=0.09$ [48].

Since the assumption of fast chemistry does not always hold, the reaction rates can also be limited by chemical kinetics (CKI):

$$
R R_{r}^{\mathrm{CKI}}=k_{f r} \prod_{k=1}^{N_{k}} c_{k}^{v_{k r}^{\prime}}-k_{b r} \prod_{k=1}^{N_{k}} c_{k}^{v_{k r}^{\prime \prime}},
$$

where $c_{k}$ is the filtered concentration of species $k$, and $k_{f r}$ and $k_{b r}$ are the forward and backward rate constants, respectively [54]. The rates of the reactions Eq. (3) and (4) are computed as Arrhenius functions, where the pre-exponential factors are adjusted in a way such that the local flame speed is calculated according to the local equivalence ratio as shown in detail by Franzelli et al. [6].

The overall reaction rate is then computed as the minimum of the EDM and the CKI reaction rates according to

$$
R R_{r}^{\mathrm{EDM} / \mathrm{CKI}}=\min \left(R R_{r}^{\mathrm{EDM}}, R R_{r}^{\mathrm{CKI}}\right) .
$$

For the operating condition studied in this work, the $\mathrm{CH}_{4}$ oxidation Eq. (3) is firstly limited by $R R_{r}^{\mathrm{CKI}}$ until the mixture reaches a temperature of $T \approx 1100-1500 \mathrm{~K}$, and afterwards the reaction is controlled by mixing, i.e., by the EDM rate $R R_{r}^{\mathrm{EDM}}$. The $\mathrm{CO} \rightleftharpoons \mathrm{CO}_{2}$ reactions Eq. (4) on the other hand are generally largely controlled by the EDM rate as they take place in high temperature regions.

\subsubsection{FRC Model}

In the second combustion model with finite rate chemistry (FRC), methane combustion reactions are modeled by the skeletal DRM19 reaction scheme [55]. DRM19 is a reduced reaction mechanism based on the GRI-Mech 1.2 [56] and involves 19 chemically active species and 84 reactions. Thus, 19 additional transport equations were solved for the reaction species mass fractions. To overcome the stiff coupling of these transport equations, species transport and enthalpy equations are integrated implicitly as a coupled set of equations. This is embedded in the fractional step method SICS which uses an operator splitting approach to integrate the full set of governing equations for reacting flows.

In the FRC model, subgrid-scale turbulence-chemistry interaction (TCI) is included using an assumed PDF model (APDF) following Girimaji [57]. Thereby additional transport equations are solved for the temperature variance and the sum of species 
variances as described in detail by Gerlinger [38]. The reaction rate is determined by

$$
R R_{r}^{\mathrm{FRC}-\mathrm{APDF}}=\int_{-\infty}^{\infty} R R_{r}^{\mathrm{FRC}}\left(\hat{T}, \hat{Y}_{i}\right) P(\hat{T}) P\left(\hat{Y}_{i}\right) d \hat{T} d \hat{Y}_{i},
$$

where $T$ is the temperature, $Y$ is the mass fraction vector, $P$ is a PDF, () denotes a random variable and $R R_{r}^{\mathrm{FRC}}$ is the FRC reaction rate. The PDFs of temperature and mass fractions are modeled as clipped Gaussian and multi-variate Beta-PDF types, respectively [38, 58, 57].

A recent study has examined the contributions of resolved and modeled fluctuations for the present FRC-APDF simulation [59]. It was found that within the flame zone, more than $95 \%$ of the variance of mass fractions and of turbulent kinetic energy (see also Fig. 2b), and more than $90 \%$ of the temperature variance are resolved. A comparison of simulations with and without subgrid model further showed that the effect of the subgrid APDF model is small as expected from the large fraction of resolved fluctuations. Nevertheless the simulation with the subgrid APDF model yielded a slightly better agreement with experimental data, despite the simplifying assumption that fluctuations of temperature and mass fractions are uncorrelated.

Using the FRC model without subgrid APDF model does not imply that TCI of the flame is neglected at all. In principle, only the non-linear part of the subgrid-TCI is neglected. In the SAS computation using the FRC model without subgrid APDF model described in Ref. [59], most of the experimentally measured temperature variance and species variances were resolved by the simulation. Hence TCI can be assumed to be resolved to a high extent as well. Although the FRC model is more expensive than other models, it is versatile and intrinsically includes various impacts on combustion such as, e.g., strain rate and heat loss.

\subsection{Boundary conditions}

The mass fluxes at the air and fuel inlets are set to the values presented in Table 1. In the measurements, the temperature of fresh gas entering the combustion chamber was found to vary between 320 and $380 \mathrm{~K}$ due to the contact with the hot walls of the test rig [12]. This preheating was considered in the previous numerical study of Franzelli et al. [6] by assuming an inflow temperature of fuel and air of $320 \mathrm{~K}$, which equals the minimum of the measured fresh gas temperature. In this work we follow Franzelli et al. to have a good comparability with their study. However, this leads to a slight underestimation of the temperatures within the combustion chamber. Furthermore, walls are assumed to be adiabatic which is also in accordance with the study of Franzelli et al.

Besides the hydrodynamic boundary conditions described above, acoustic boundary conditions are of great importance for the prediction of thermoacoustic instabilities. In the present work Time Domain Impedance Boundary Conditions (TDIBC) $[60,27,28,61,62]$ are used to apply the complex boundary reflection coefficient

$$
R(z)=\frac{G(z)}{F(z)}=\frac{b_{0}+b_{1} z^{-1}+\ldots b_{M} z^{-M}}{1+a_{1} z^{-1}+\ldots a_{N} z^{-N}}
$$

where $R$ is the reflection coefficient, $F$ is the incident and $G$ is the reflected acoustic wave, $b_{i}$ and $a_{i}$ are model constants and $M$ and $N$ are the orders of the nominator and the denominator, respectively. Moreover, $z=e^{i \omega}$ where $i$ is the imaginary unit and $\omega$ is the circular frequency. The model constants $a_{i}$ and $b_{i}$ are computed by fitting the general ansatz function Eq. (9) to a target function as described by Kaess et al. [28]. The frequency response Eq. (9) is then transformed into the time domain using an inverse z-transform. Details of this transformation, and of the implementation and performance of TDIBC in the DLR THETA code can be found in Ref. [37].

The atmospheric combustor outlet is modeled as an acoustically open end, which has been characterized using analytical and numerical methods $[63,64]$. In this work the TDIBC ansatz function Eq. (9) with order $M=45$ and $N=0$ is fitted to the analytical solution of Munt [63]. Figure 3 presents the analytical reflection coefficient and the fitted TDIBC function. The reflection coefficient computed with TDIBC and the analytical solution agree very well with respect to both the modulus and the phase shift. The computational domain further includes an air inlet tube ( $d=25 \mathrm{~mm}, l=50 \mathrm{~mm}$ ) upstream of the plenum with a fully reflecting acoustic boundary condition at its upstream end in accordance with the choked orifice plate in the experiment specified in Sect. 2.2. Fuel is supplied into the fuel plenum through a circular orifice $(d=6 \mathrm{~mm})$ in the plenum wall, which is also modeled as a fully reflecting acoustic boundary.

\section{Results and discussion}

\subsection{Flow field, mixture fraction and temperature}

In the following, the fields of velocity, mixture fraction and temperature obtained with EDM and FRC are compared to the experimental values of Meier et al. [12] and to the LES of Franzelli et al. [6]. For all quantities Reynolds averaging is applied. RMS values for the simulations represent the resolved fluctuations (as noted in Sect. 3.3.2, the ratio of resolved to total variance in the considered domain is $>0.95$ for the turbulent kinetic energy and mass fractions, and $>0.9$ for temperature). Figure 4 shows the mean axial and radial velocity profiles at downstream positions of $h=1.5,5,15,25$ and $35 \mathrm{~mm}$. For both the EDM and the FRC combustion model, the velocity profiles computed within this work agree well with both the experimental data and the LES results of Franzelli et al. As can be seen from Fig. 4a, the spread of the swirling jet and the location and strength of the inner (IRZ) and outer recirculation zone (ORZ) are met accurately.

The computed mixing of fuel and air is assessed in Fig. 5 by means of mixture fraction profiles at $h=6,10,20,30$ and 60 $\mathrm{mm}$. For both experiment and simulations, the mixture fraction was determined from the mass fractions of $\mathrm{C}, \mathrm{H}$ and $\mathrm{O}$ elements according to the definition of Bilger et al. [65]. The mass fractions of $\mathrm{C}, \mathrm{H}$, and $\mathrm{O}$ were obtained from the full set of chemical species present in the numerical reaction mechanism or experimental data, respectively. For both the EDM and FRC model, the calculated mean mixture fraction are notably lower than the 

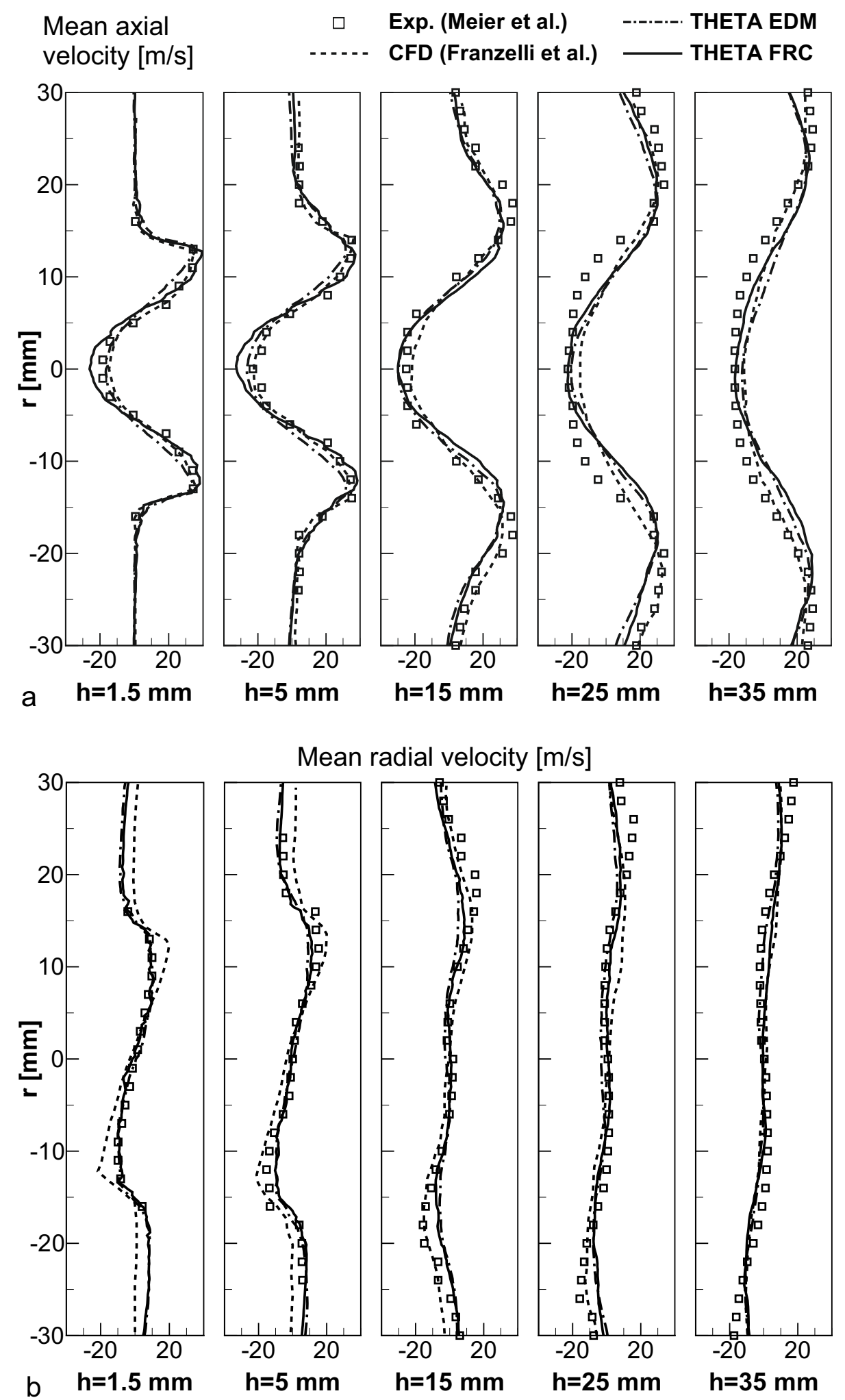

Figure 4: Profiles of (a) mean axial velocity and (b) mean radial velocity at different axial positions. 

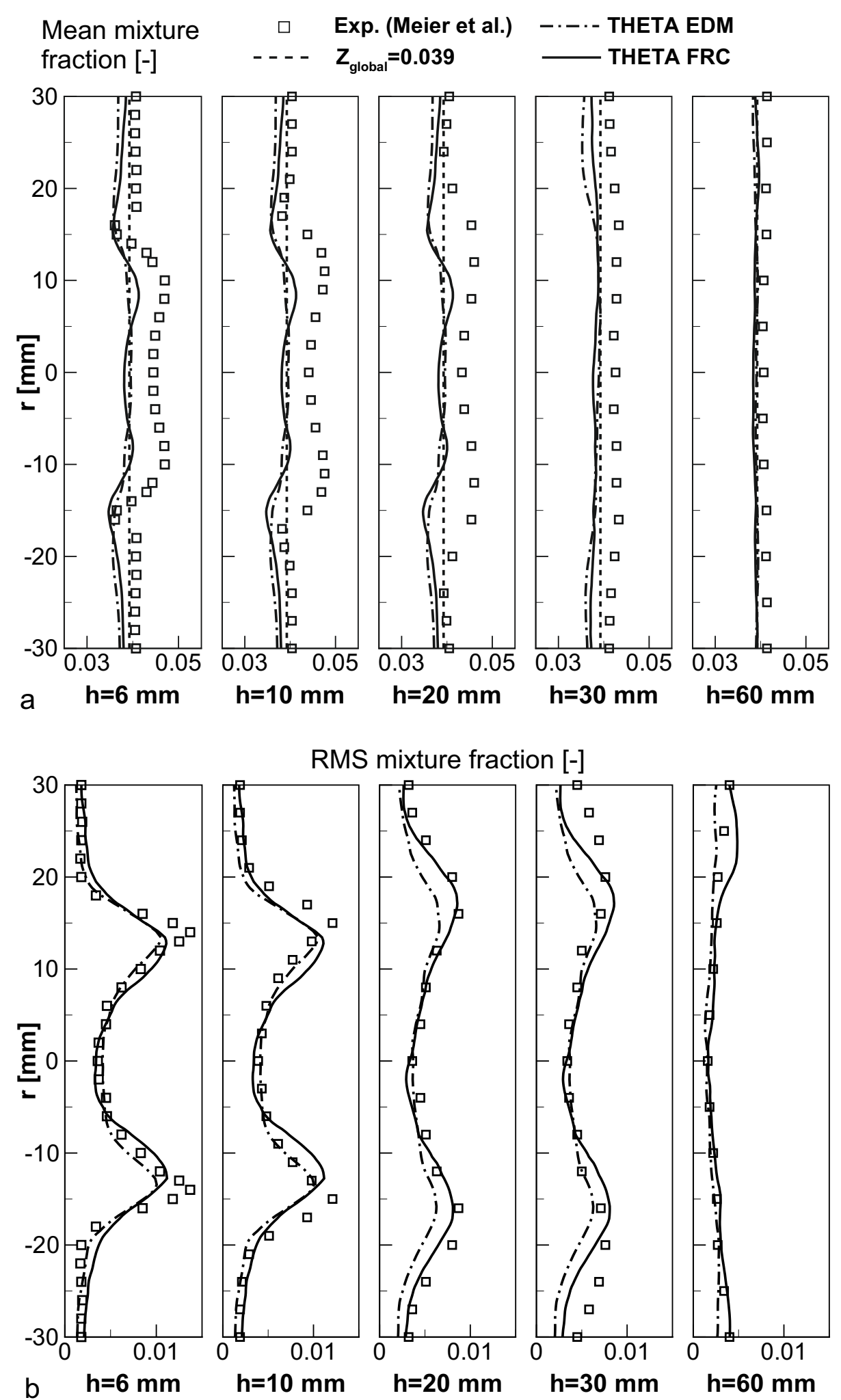

Figure 5: Profiles of (a) mean and (b) RMS mixture fraction at different axial positions. 

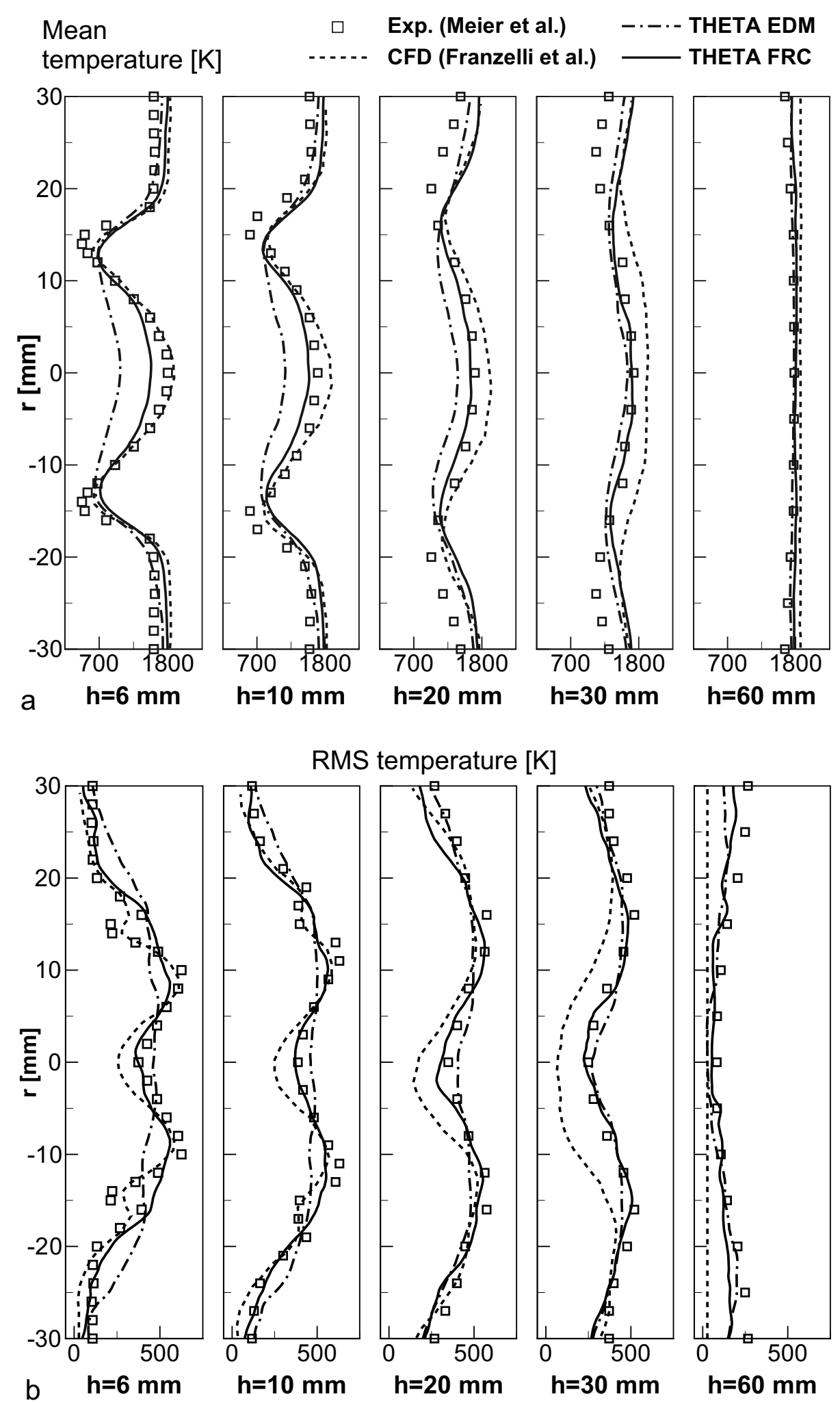

Figure 6: Profiles of (a) mean and (b) RMS temperature at different axial positions. 
measured values at the axial positions of $h \leq 30 \mathrm{~mm}$. At the most downstream position $h=60 \mathrm{~mm}$ the calculated mean mixture fraction agrees well with the global mixture fraction. Since the calculated mean velocity fields discussed previously do agree well with the experiments, the difference in mean mixture fraction cannot be caused by different convective transport of the fuel within the combustor. The underprediction of the mean mixture fraction is therefore attributed to measurement uncertainties. It is noted that Meier et al. [12] point out that the measured mixture fractions are by trend biased towards higher values especially in low-temperature regions. Unlike the mean values of mixture fraction, the calculated and measured RMS mixture fraction profiles agree well. The amplitude of equivalence ratio fluctuations which contribute to the driving of the thermoacoustic oscillation is therefore accurately reproduced.

Figure 6a presents mean temperature profiles for $h=6,10$, 20, 30 and $60 \mathrm{~mm}$. Generally the temperatures computed with EDM and FRC agree well with both the experimental data and the LES of Franzelli et al. For all models, lower temperatures compared to experiment are observed in the ORZ $(h \leq 30 \mathrm{~mm}$, $|r|>20 \mathrm{~mm}$ ), which is attributed to heat loss at the chamber walls. At the combustion chamber inlet $(h \leq 10 \mathrm{~mm}$, $|r|<15 \mathrm{~mm}$ ), however, deviations between EDM and FRC calculations are visible. With the EDM model the mean lift-off height of the flame is overpredicted compared to the measurements, probably due to the simplified chemistry model. However, these deviations are limited to a comparably small region ( $h \leq 10 \mathrm{~mm}$ and $r \leq 10 \mathrm{~mm}$ ) that is smaller than $1 \%$ of the combustion chamber volume. Since the mean temperature is predicted well in most other regions of the combustion chamber, the temperature deviations visible for the EDM calculations have only a minor influence on the combustor acoustics as shown in Sect. 4.3. Apart from the lift-off height, the predictions of the simple EDM model are remarkably good, which is attributed to CKI extension based on local equivalence ratio (Sect. 3.3.1). With the FRC model, by contrast, also the liftoff height of the flame is well predicted. This is reasonable since the flame base of a lifted turbulent swirl flame is a zone of strong unsteady turbulence-chemistry interaction $[66,67]$ that can be better resolved by the detailed FRC model. A similar behavior at the flame base was seen in a recent comparison of LES with and without subgrid modeling of turbulence-chemistry interaction by See and Ihme [68].

Figure $6 \mathrm{~b}$ shows RMS temperature profiles within the combustion chamber. The data set calculated with FRC agrees very well with the experimental data at all plotted positions. The results of Franzelli et al. also agree well, except for the area around the central axis $(r=0)$ at $h \leq 30 \mathrm{~mm}$, where the RMS values are too low. Regarding the EDM results, the RMS temperature profiles agree well with the experimental data at the three downstream positions ( $h \geq 20 \mathrm{~mm}$ ). At the two most upstream positions $h=6$ and $10 \mathrm{~mm}$, a reasonable agreement between EDM calculations and the measured results is visible. As for the flame lift-off height discussed above, this deviation of the RMS temperatures likely results from the simplified modeling of unsteady turbulence-chemistry interaction with EDM.

\subsection{Progress of reaction and mixing}

The thermochemical states within the combustion chamber are analyzed by means of scatter plots of temperature vs. mixture fraction at $h=6 \mathrm{~mm}$ shown in Fig. 7a-c. In the experimental data the mixture fraction varies in the range $0.015 \leq$ $Z \leq 0.08$. The premixing achieved within the swirler is thus not perfect. Within the IRZ $(r=0-6 \mathrm{~mm})$ the samples are primarily at adiabatic flame temperature, but there are also several samples visible that correspond to unburned gas or partially reacted gas mixtures. These likely stem from fresh gas entrained into the IRZ that has partly mixed with burned gas but not yet reacted due to delays caused by finite rate chemistry [12]. In the ORZ ( $r=18-30 \mathrm{~mm}$ ), by contrast, reaction is virtually completed and the temperature is on average around $200 \mathrm{~K}$ lower than the adiabatic flame temperature. This temperature reduction is likely caused by heat loss at the side walls or at the burner plate [12].

The scatter plot obtained with the FRC model shown in Fig. $7 \mathrm{~b}$ agrees well with the experimental data. The scattering range in mixture fraction amounts to $0.02 \leq Z \leq 0.08$ which is in good agreement with the measurements. Within the ORZ the gas is predominantly at equilibrium with temperatures close to the adiabatic flame temperature. In comparison to the experiments, the gas temperature is not reduced by heat losses since the combustion chamber walls are assumed to be adiabatic. Regarding the IRZ, the scattering in mixture fraction and temperature is in accordance with the experimental data. The intermittent entrainment and mixing of unburned gas at this position is thus predicted well by the FRC calculations.

Figure 7c presents the scatter plot computed with EDM at $h=6 \mathrm{~mm}$. In comparison to the measurements and the FRC calculations, the thermochemical gas states are more uniformly distributed between fresh gas and equilibrium states. In accordance, the clustering of gas states close to equilibrium within the IRZ and ORZ is less distinct and the flame within the IRZ is more likely to be in an intermediate than an equilibrium state for the EDM calculations. Furthermore, the mixture fraction values plotted for EDM and FRC are generally slightly lower than for the experimental data. Figure $7 d$ presents the distribution of the mixture fraction. Even though the overall shape of the calculated distributions agrees well with the measured distribution, the peaks of both THETA models and the data from Franzelli et al. appear at lower mixture fractions. This deviation is mainly attributed to measurement uncertainties, which bias the measured mixture fractions towards higher values [12].

The reaction and mixing progress within the combustion chamber is discussed by means of scatter plots at downstream positions $h=15,30,60$ and $80 \mathrm{~mm}$ shown in Fig. 8. It is seen that both mixing and reaction proceed with increasing downstream position, which is indicated by a decreasing spread of mixture fraction around the global value and an increase of temperature towards the adiabatic value, respectively. For both $\mathrm{EDM}$ and FRC calculations, the predicted reaction progress is slightly faster than in the experiment. This becomes especially apparent at the downstream position $h=60 \mathrm{~mm}$, where more intermediate states are visible in the experiment than in the cal- 

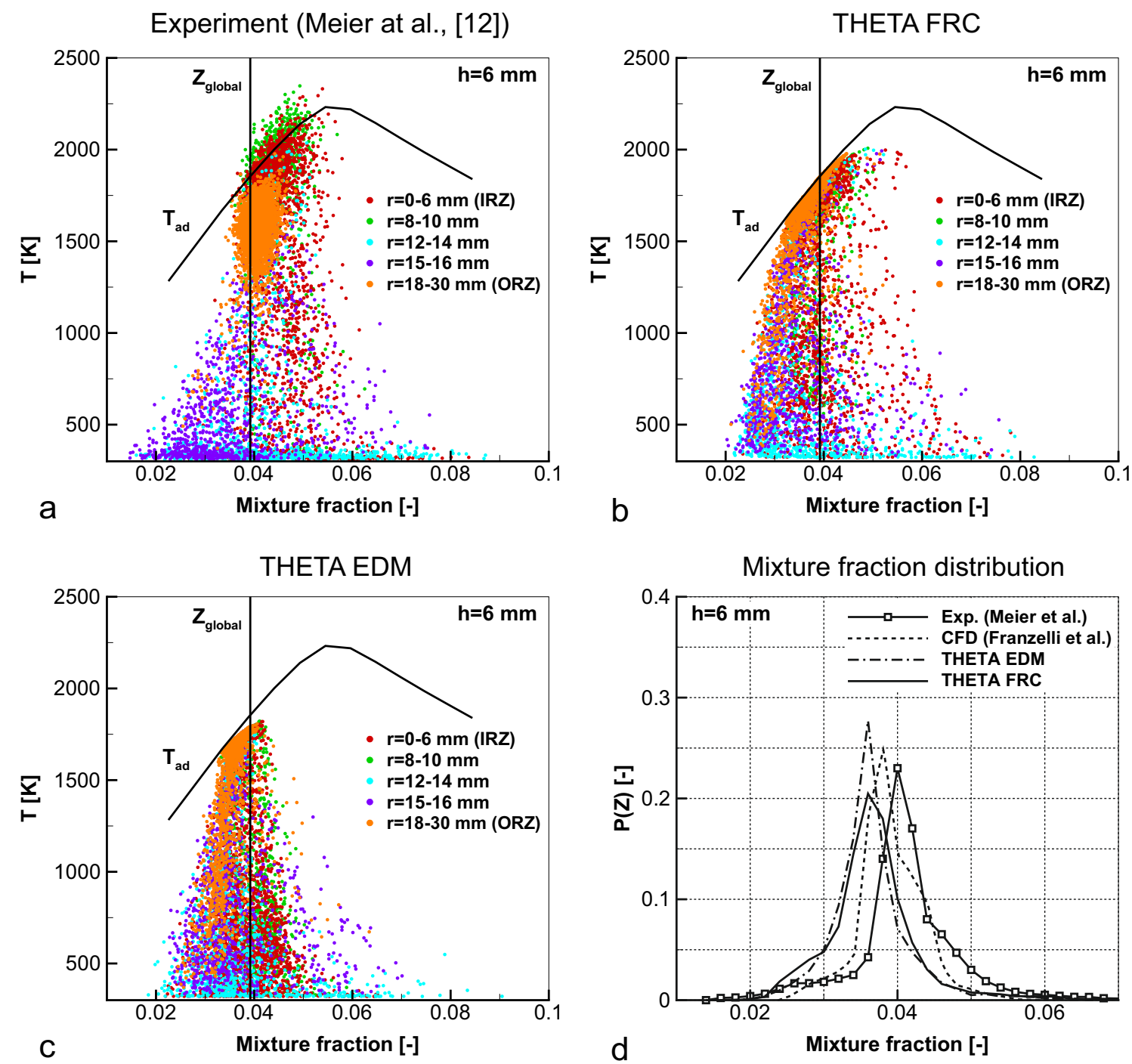

Figure 7: (a)-(c) Correlation between temperature and mixture fraction and (d) distribution of mixture fraction at $h=6 \mathrm{~mm}$.

culations. At $h=80 \mathrm{~mm}$ reaction progress is virtually complete, i.e. the gas has largely reached adiabatic temperatures for both the experiment and the computations. The scattering in temperature of the measured data at $h=80 \mathrm{~mm}$ can be related to inherent measurement uncertainties [12]. A slight underprediction of temperatures, however, is visible for EDM at $h=60$ and $80 \mathrm{~mm}$. An analysis of CO concentrations (not shown) revealed that for EDM significant levels of $\mathrm{CO}$ are still present at these positions and therefore the gas has not yet reached the fully reacted state. The presence of $\mathrm{CO}$ is caused by the relatively slow CO oxidation Eq. (4) of the two-step BFER mechanism especially for relatively fuel-rich mixtures.

The mixing progress in downstream direction computed with FRC and EDM is in good agreement with the experimental data as illustrated by Fig. 8, even though the mixing is slightly faster in case of the EDM calculations. Even at the last downstream position $h=80 \mathrm{~mm}$, mixing is not fully completed as indicated by the scattering in mixture fraction. In case of the calculations, the mean mixture fraction at this position equals the global mixture fraction of the analyzed operating conditions as expected. As mentioned above, a slight bias of the experimental data set towards higher mixture fraction values is observed at this location.

\subsection{Thermoacoustic oscillation}

\subsubsection{Power spectral densities}

Figure 9 compares power spectral densities (PSD) for the simulations with EDM and FRC with the experimental data for the configuration with quartz windows (see Sect. 2.2). For both EDM (90 ms runtime) and FRC (75 ms runtime), two overlapping pressure time-series of $60 \mathrm{~ms}$ were generated in order to have the same spectral resolution, i.e., 16.7 Hz. The PSDs shown in Figs. 9 and 10 are interpolated averaged Fourier transforms of these two overlapping signals. The experimental spectra are averages of time-series of $1 \mathrm{~s}$, corresponding to a spectral resolution of $1 \mathrm{~Hz}$. 
Exp. (Meier et al.)
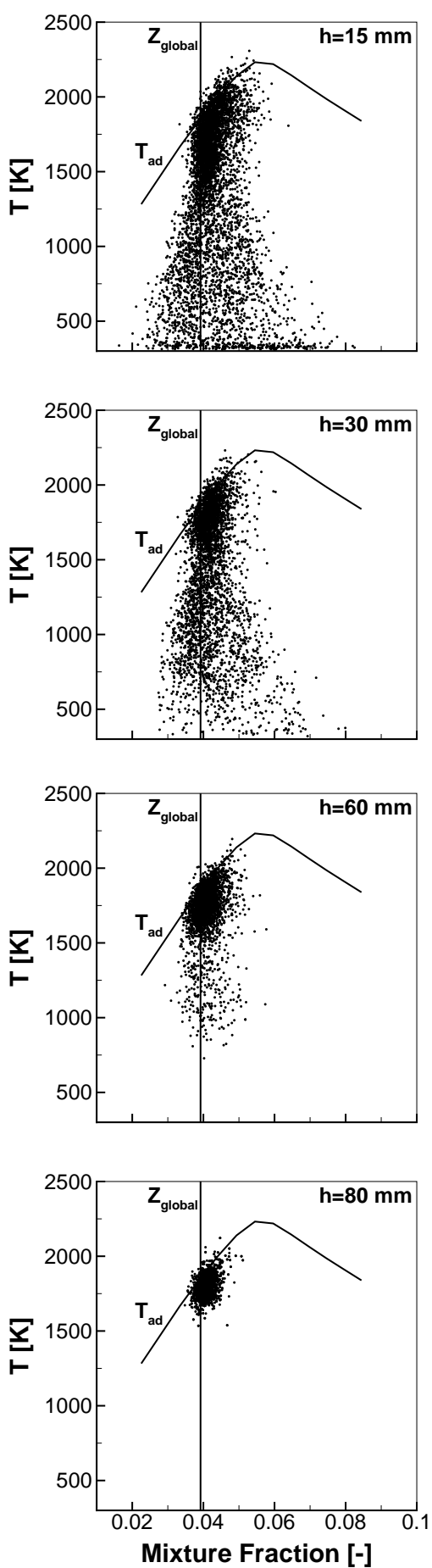

THETA FRC
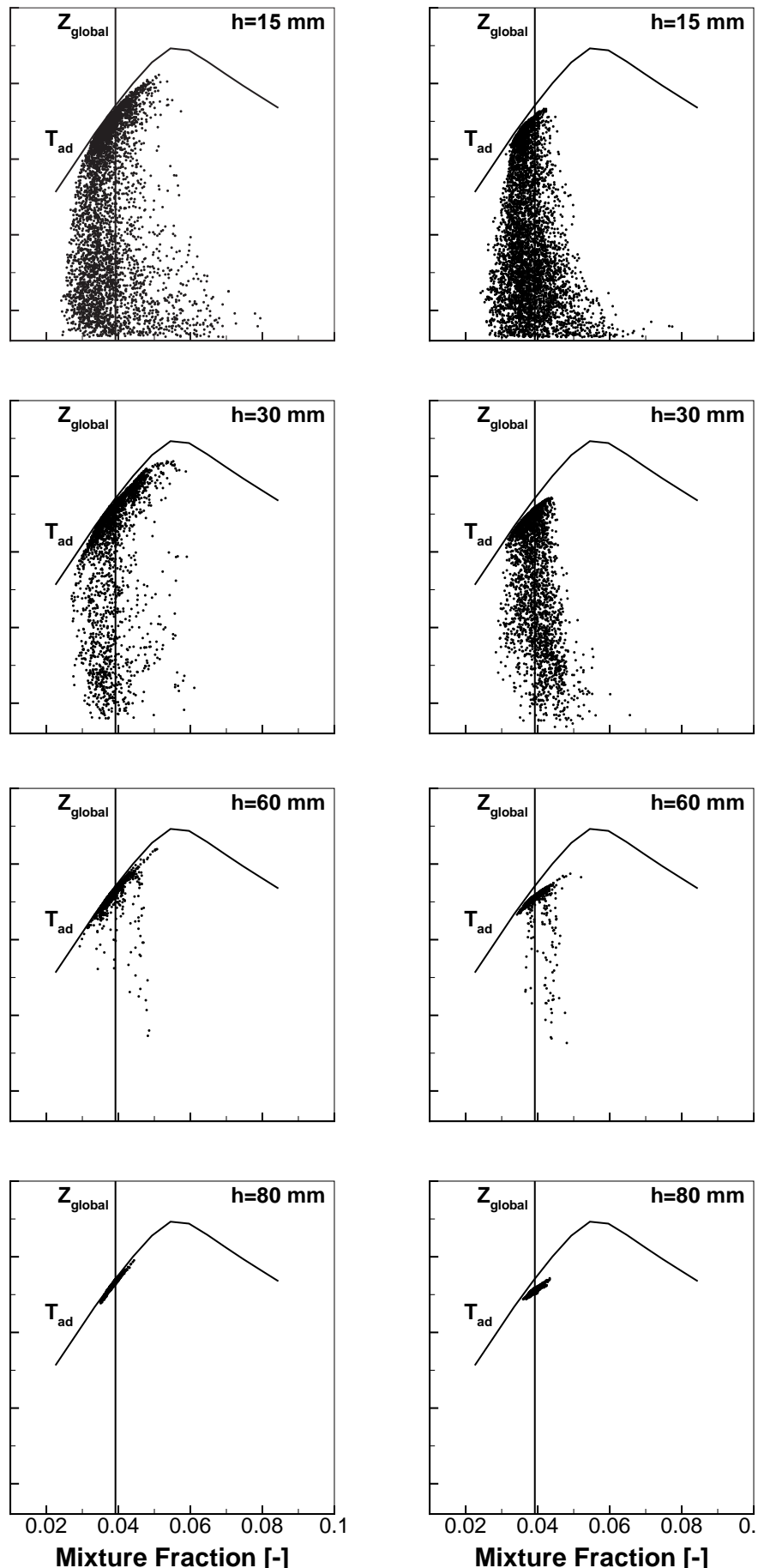

Figure 8: Correlation between temperature and mixture fraction at different downstream positions. 

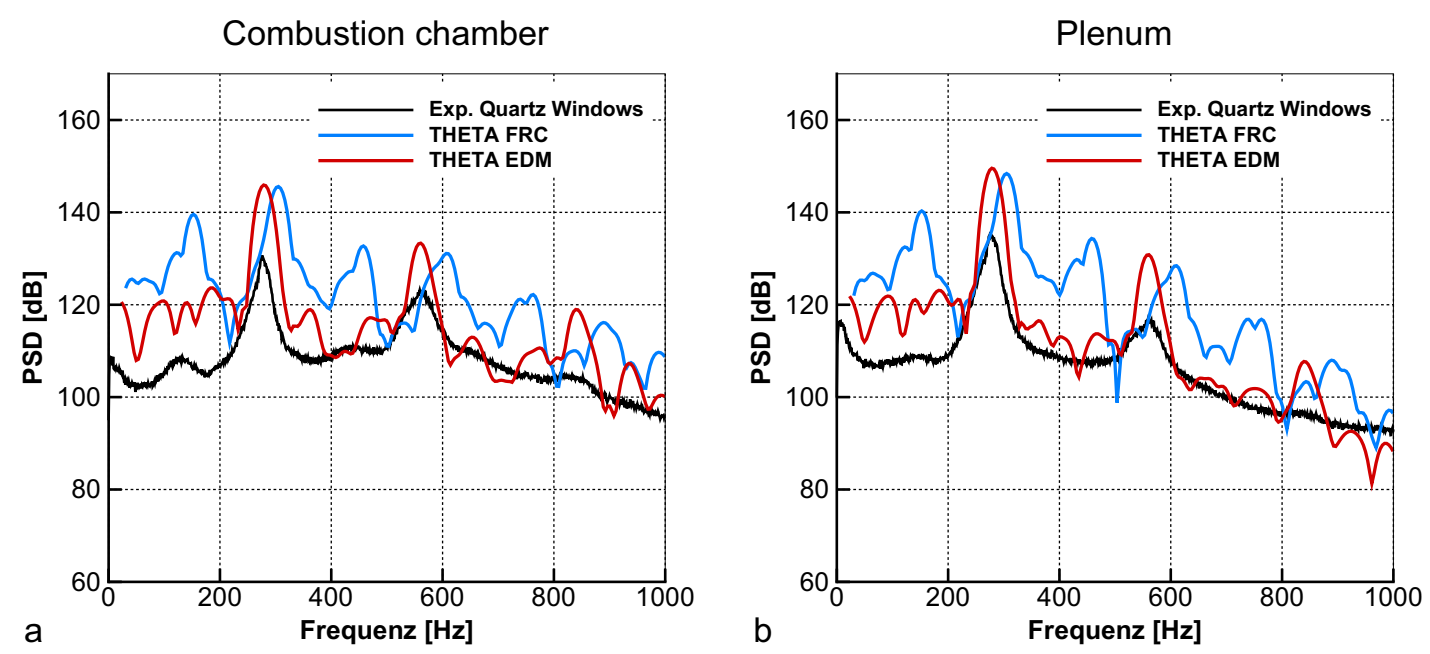

Figure 9: Power spectral densities from experiments with quartz windows and from computations with THETA EDM and FRC.
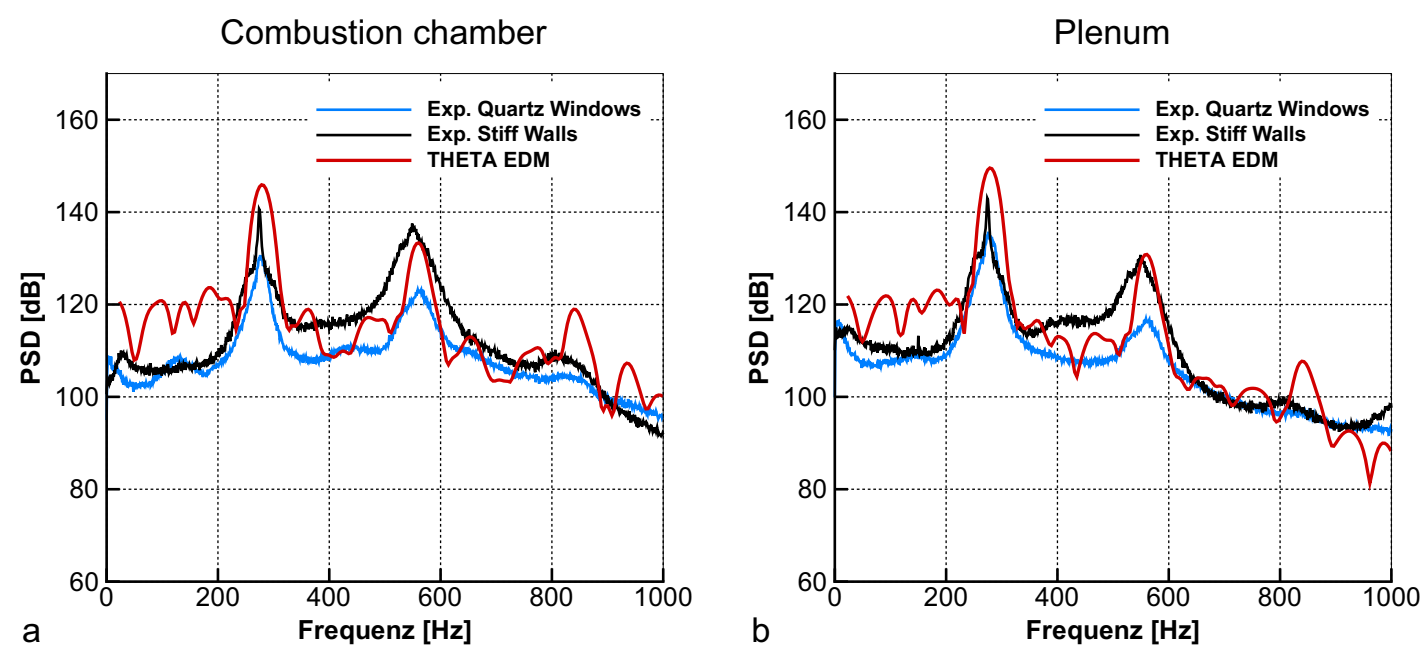

Figure 10: Influence of acoustic damping at the combustion chamber walls on the power spectral densities.

For the experimental spectrum in the combustion chamber, the highest PSD of approximately $130 \mathrm{~dB}$ is measured at a frequency of around $275 \mathrm{~Hz}$, which corresponds to the main thermoacoustic oscillation of the burner. Furthermore, a second peak of about $123 \mathrm{~dB}$ is visible at the first harmonic frequency of $550 \mathrm{~Hz}$. These two maxima are also identified in the PSD of the air plenum, where the signal at the thermoacoustic frequency is around $3 \mathrm{~dB}$ higher than within the combustion chamber.

With EDM, the thermoacoustic frequency is predicted to around $290 \mathrm{~Hz}$, which is about $15 \mathrm{~Hz}$ higher compared to the experiment. The amplitudes of the predicted oscillation at $290 \mathrm{~Hz}$ within the combustion chamber and the plenum are $146 \mathrm{~dB}$ and $150 \mathrm{~dB}$, respectively. In comparison to the experimental data, this amplitude is hence overpredicted by $16 \mathrm{~dB}$ within the combustion chamber and by $17 \mathrm{~dB}$ in the plenum. For the FRC model, the thermoacoustic frequency is computed to $300 \mathrm{~Hz}$ and is hence overpredicted by $25 \mathrm{~Hz}$ compared to the experimental data. The thermoacoustic amplitude amounts to $146 \mathrm{~dB}$ within the combustion chamber, which is $16 \mathrm{~dB}$ higher than the measured amplitude.

The relatively small differences to the experimental thermoacoustic frequency for EDM and FRC of $15 \mathrm{~Hz}$ and $25 \mathrm{~Hz}$, respectively, are probably related to minor deviations of temperature and progress of mixing and reaction discussed in Sects. 4.1 and 4.2. These deviations, however, can hardly explain the strong overprediction of the thermoacoustic amplitude of about $16 \mathrm{~dB}$ for both EDM and FRC. To elucidate the reasons for this overprediction, possible damping mechanisms in the model combustor are examined in the next subsection. 
The PSD computed with FRC further exhibits additional peaks at the subharmonic frequency of $150 \mathrm{~Hz}$ and its multiples at 450 and $750 \mathrm{~Hz}$. The subharmonic frequency of $150 \mathrm{~Hz}$ also appears with lower amplitude in the experiment, whereas no significant subharmonic peaks appear for EDM. In the experiments the subharmonic oscillation was very unstable in the sense that its amplitude changed largely for small changes of ambient conditions. This may explain that its amplitude is also largely affected by the choice of chemistry model as seen in the comparison of spectra for FRC and EDM.

\subsubsection{Damping effects of combustion chamber side walls}

Acoustic damping in gas turbine typical combustion chambers is commonly attributed to boundary layer losses, to interaction of sound with turbulent flow and to radiation of acoustic energy across the combustor boundary surfaces. In the experimental studies of the PRECCINSTA combustor, an additional origin of acoustic damping is present: as described in Sect. 2.2, the standard configuration of the combustor uses quartz windows as side walls of the combustion chamber, which are loosely mounted and therefore may damp acoustic oscillations. The present numerical model considers acoustic losses at the outflow by means of impedance boundary conditions, but all other boundaries of the numerical domain including the side walls are treated as acoustically hard.

In order to estimate the amount of damping caused by the loose side walls, additional acoustic measurements were performed using a modified combustion chamber with rigid metal walls where the damping at the walls is largely suppressed (see Sect. 2.2). Figure 10 compares the measured PSDs of the standard and the modified configurations of the PRECCINSTA burner. In the modified configuration with stiff combustor walls, the thermoacoustic amplitude is now around $10 \mathrm{~dB}$ higher than with the standard configuration with quartz windows embedded in the side walls. In contrast, the frequency of the thermoacoustic oscillation is not altered between both configurations. The comparison of the PSD obtained with THETA EDM with that from the modified combustor configuration in Fig. 10 shows that the amplitude of the thermoacoustic oscillation is now only overpredicted by about $5 \mathrm{~dB}$ in the combustion chamber and the air plenum. This demonstrates that damping at side walls can be an important effect in experimental tests of combustion instabilities that should be taken into account in the validation of numerical models.

\subsection{Oscillations of velocity and equivalence ratio in the burner nozzle}

While in perfectly premixed combustors thermoacoustic oscillations are mainly driven by velocity oscillations at the burner nozzle, partially premixed combustors exhibit a second source of excitation, namely the oscillation of equivalence ratio [13]. Generally the oscillations of velocity and equivalence ratio exhibit a certain phase difference [12, 6, 8, 16], and the resulting oscillation of heat release is largely affected by the corresponding time-delay $[69,15,16]$. Usually this delay is caused by different acoustic impedances of fuel and air supply in combination with convective delays in the burner nozzle. Since

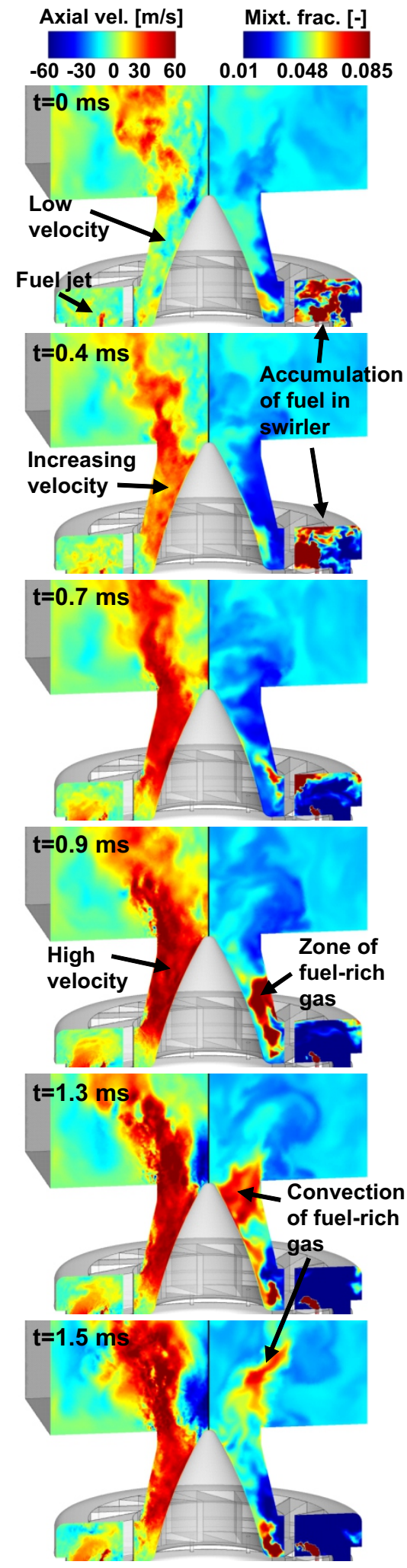

Figure 11: Temporal variations of axial velocity (left) and mixture fraction (right). 

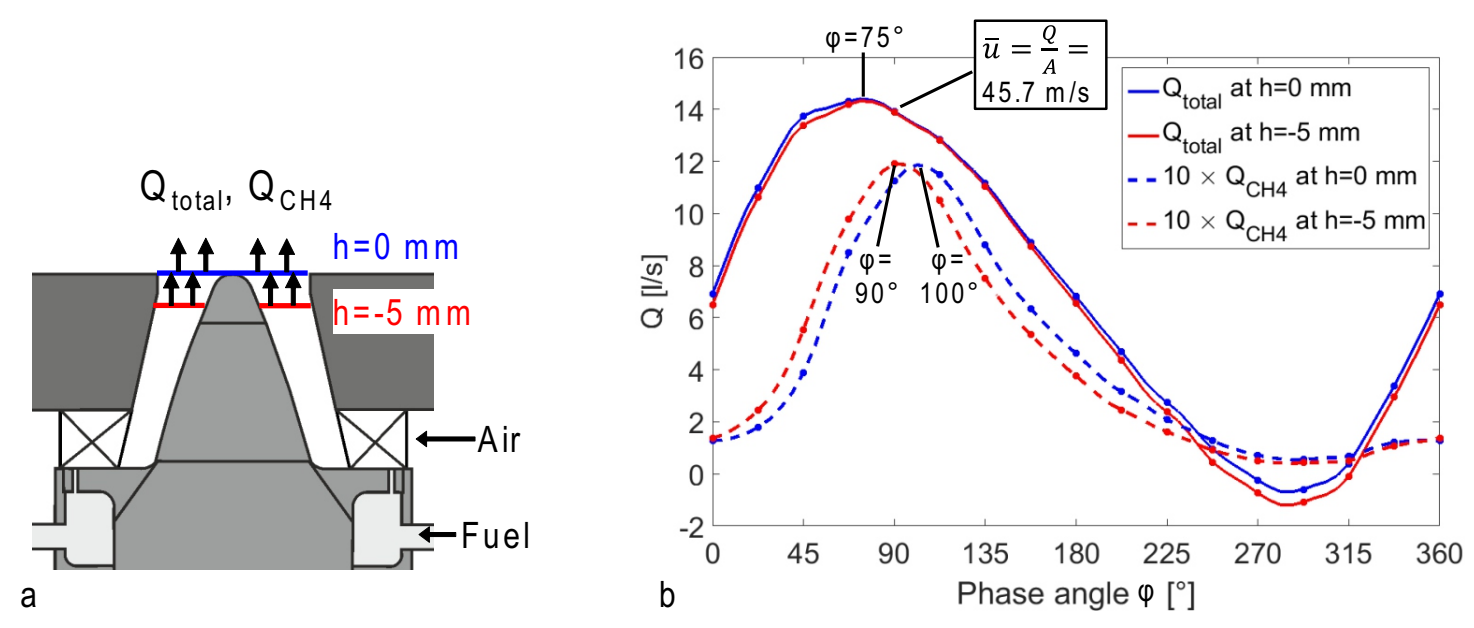

Figure 12: a) Calculation of total volumetric flux $Q_{\mathrm{total}}$ and volumetric flux of fuel $Q_{\mathrm{CH} 4}$ at $h=0 \mathrm{~mm}$ and $h=-5 \mathrm{~mm}$. b) Phase averages of $Q_{\mathrm{total}}$ and $Q_{\mathrm{CH} 4}$ (scaled by a factor of 10).

this region of the combustor is hardly accessible by experiment, the present $3 \mathrm{D}$ time-resolved computations provide a valuable means for analyzing the formation and interplay of velocity and equivalence ratio oscillations and their effect on combustion instability.

Figure 11 shows a time-series of axial velocity and mixture fraction in the central plane through the combustor from the calculation with EDM (results for FRC are equivalent since modeling of non-reacting flow and transport is identical for EDM and FRC). The pressure oscillations in the chamber and plenum lead to an oscillation of axial velocity in the nozzle. At $t=0 \mathrm{~ms}$, it is seen that the axial flow in the nozzle is almost stagnant, i.e., strongly reduced compared to the average flow velocity. The jet of fuel in the swirler, on the other hand, is relatively weakly affected by the pressure oscillations due to the high impedance of the thin fuel channels, and therefore fuel accumulates in the swirler as seen at $t=0 \mathrm{~ms}$ and $t=0.4 \mathrm{~ms}$. The axial flow oscillates in phase at different heights within the nozzle. It starts increasing at $t=0.4 \mathrm{~ms}$ and reaches its maximum between $t=0.9 \mathrm{~ms}$ and $t=1.3 \mathrm{~ms}$. The zone of fuel-rich gas that accumulated in the swirler is thereby convected into the chamber from $t=0.9 \mathrm{~ms}$ until $t=1.5 \mathrm{~ms}$. While the maximum axial velocity at the chamber inlet ( $h=0 \mathrm{~mm}$ ) occurs at $t \approx 1.1 \mathrm{~ms}$, the equivalence ratio at the inlet reaches its maximum at $t \approx 1.3 \mathrm{~ms}$, i.e., about $0.2 \mathrm{~ms} \hat{=} 20^{\circ}$ later. The results thereby reveal that the time-delay between the oscillations of velocity and equivalence ratio at the chamber inlet is caused by the combination of a relatively constant rate of fuel injection in the swirler and a strongly oscillating flow in the swirl vanes and the burner nozzle.

For a quantitative analysis of the delay between oscillations of velocity and equivalence ratio, the total volumetric flux $Q_{\text {total }}(t)$ and the volumetric flux of fuel $Q_{\mathrm{CH} 4}(t)$ are calculated at the exit of the burner nozzle at heights $h=0 \mathrm{~mm}$ and $h=-5$ $\mathrm{mm}$ as shown in Fig. 12a. The oscillations of $Q_{\text {total }}(t)$ and $Q_{\mathrm{CH} 4}(t)$ are then phase-averaged with respect to the angle $\varphi$ of the thermoacoustic oscillation (determined from $Q_{\text {total }}(t)$ at $h=0 \mathrm{~mm}$ ). The resulting phase-averaged variations plotted in Fig. 12b show that $Q_{\text {total }}(\varphi)$ at $h=0 \mathrm{~mm}$ and $h=-5 \mathrm{~mm}$ oscillate in phase and both reach a maximum at $\varphi=75^{\circ}$. The fact that the oscillations of $Q_{\text {total }}(\varphi)$ at different heights are in phase, which was also noted above in the discussion of Fig. 11 , indicates that they are not driven by convection, but by an acoustic eigenmode. The fluxes of fuel, by contrast, are delayed and reach their maximum at $\varphi=90^{\circ}$ for $h=-5 \mathrm{~mm}$ and $\varphi=100^{\circ}$ for $h=0 \mathrm{~mm}$. For a $290 \mathrm{~Hz}$ oscillation, the delay of $10^{\circ}$ between $h=-5$ and $h=0 \mathrm{~mm}$ corresponds to $\Delta t=0.1$ ms. This agrees well to the corresponding convective delay of $\Delta t=\frac{\Delta h}{\bar{u}}=0.11 \mathrm{~ms}$, obtained for an axial velocity of $\bar{u}=45.7$ $\mathrm{m} / \mathrm{s}$ at $\varphi=90^{\circ}$ (see Fig. 12b) and $\Delta h=5 \mathrm{~mm}$. This confirms the conclusion drawn in the previous paragraph that the delay of equivalence ratio is caused by convection of fuel from the fuel injector in the swirler.

At $h=0 \mathrm{~mm}$, the delay between $Q_{\text {total }}$ and $Q_{\mathrm{CH} 4}$ is about $\Delta \varphi=25^{\circ}$, which corresponds to $\Delta t=0.24 \mathrm{~ms}$ for $f=290 \mathrm{~Hz}$. In a recent experimental study of a thermoacoustic oscillation in the same combustor for an operating condition of $P_{\mathrm{th}}=15 \mathrm{~kW}$ and $\phi=0.8$, the delay between $Q_{\text {total }}$ and $Q_{\mathrm{CH} 4}$ was determined as $\Delta t \approx 0.5 \mathrm{~ms}$ [16]. Assuming that $\Delta t$ is a convective delay that scales with the average flow velocity in the nozzle, the delay of $\Delta t=0.24 \mathrm{~ms}$ of the present numerical study is multiplied by the ratio of average velocity with respect to the experiment of $\frac{P_{\mathrm{th}, \mathrm{SAS}}}{P_{\mathrm{th}, \mathrm{Exp}}} \frac{\phi_{\mathrm{Exp}}}{\phi_{\mathrm{SAS}}}=\frac{25.1}{15} \frac{0.8}{0.7}=1.91$. The delay then equals to $0.46 \mathrm{~ms}$, and is thus in good agreement with the experimental value. This suggests that a similar mixing dynamics as seen in Fig. 11 also takes place in the experiment, yet at a slower time scale.

The response of the flame to the variations of axial velocity and equivalence ratio is shown in the time-series in Fig. 13, which corresponds roughly to one thermoacoustic cycle. While the zone of fuel-rich gas, visualized by a mixture fraction isosurface, is convected into the chamber $(t=0.9 \mathrm{~ms})$, few and relatively straightened reaction zones are visible and the overall heat release is near its minimum. At $t=1.5 \mathrm{~ms}$, the zone of fuel-rich gas reaches the flame zone, and at $t=2.1-3.1 \mathrm{~ms}$, the flame responds to the increased supply of fuel with an in- 


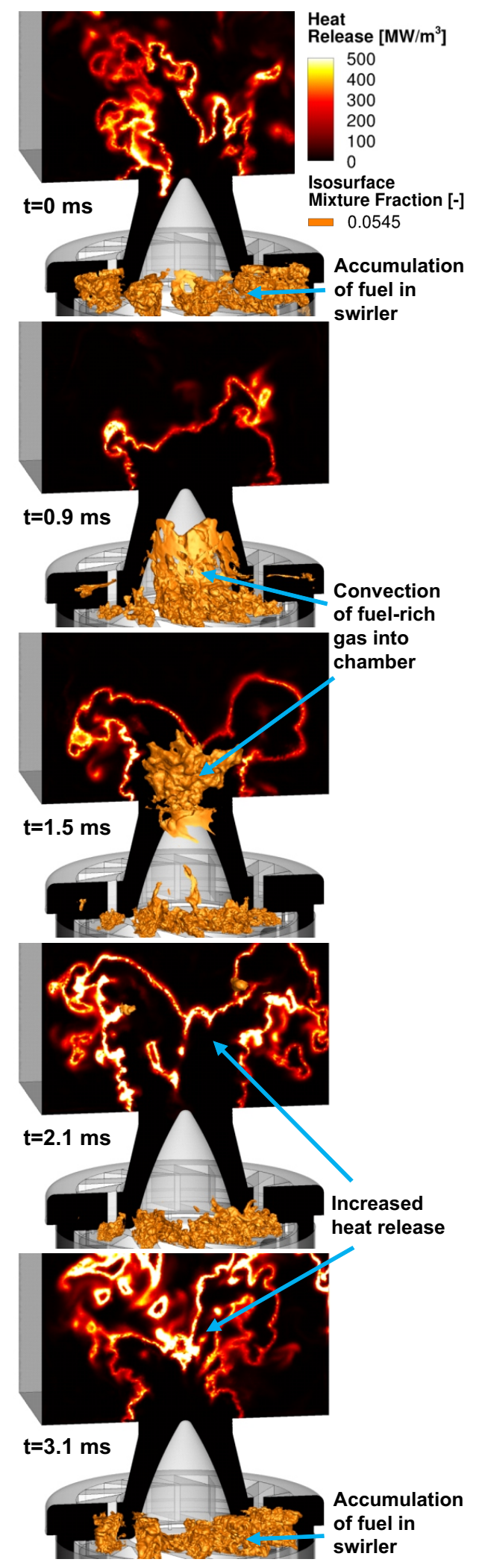

Figure 13: Temporal variations of mixture fraction and heat release. tensified heat release. This leads to an increased pressure in the combustion chamber, which slows down the flow in the burner nozzle such that fuel again accumulates in the swirler vanes, and another similar thermoacoustic cycle begins.

\section{Conclusions}

The thermoacoustic instability of a lean partially premixed flame in the PRECCINSTA swirl burner has been examined by means of CFD and experiments. Based on the suggestion from previous CFD work on this flame by Franzelli et al. [6], the channels and the plenum of the fuel supply were included in the numerical domain in order to resolve the impedance of the fuel injection jets. Turbulent fluctuations were modeled using a Scale Adaptive Simulation (SAS) technique with fine spatial resolution in the combustion regions, and the impedance of the combustor outlet surface was modeled by means of time domain impedance boundary conditions. The latter eliminates the need for a computational domain downstream of the combustor exit, and thus significantly reduces the computational cost. Two combustion models were applied, a simple Eddy Dissipation Model (EDM) and a complex detailed combustion model (FRC). For both EDM and FRC the resulting fields of velocity, temperature and mixture fraction fit well to experimental data from Meier et al. [12] with a better prediction of the flame liftoff height for the FRC model. This demonstrates the good performance of the FRC model in the region near the flame base where strong unsteady turbulence-chemistry interaction takes place.

For both models the computed frequency of the limit-cycle thermoacoustic oscillation is close to the experimental value of $275 \mathrm{~Hz}$. This is a considerable improvement compared to the LES of Franzelli et al. ( $f \approx 390 \mathrm{~Hz}$ ), which is attributed to the extended model domain including the channels and the plenum of the fuel supply that was suggested by Franzelli et al. This indicates that a proper inclusion of the fuel supply is an essential requirement for accurate modeling of thermoacoustic instability in partially premixed combustors.

The amplitudes of both models, however, are significantly overestimated by about $15 \mathrm{~dB}$ in comparison to measurements. As a possible explanation for this overprediction, the damping influence of the combustor side wall mounting was analyzed. In the standard experimental configuration of the combustor, the combustion chamber side walls consisted of loosely mounted quartz glass windows that lead to a certain degree of acoustic damping. To quantify the effect of this damping on the thermoacoustic oscillation, additional acoustic measurements were carried out for a modified configuration with tightly mounted metal side walls. For this configuration, the amplitude of the acoustic oscillation is increased by around $10 \mathrm{~dB}$ and thus close to the results of the CFD. This demonstrates that damping at side walls can be an important effect in experimental tests of combustion instabilities that should be considered in the validation of numerical models.

The present flame instability is largely affected by oscillations of equivalence ratio. The CFD results were thus used to investigate the mixing of fuel and air in the swirler and the 
burner nozzle that leads to these oscillations. It was seen that during the phase of low air velocity in the burner nozzle, fuel accumulates in the swirler near the exit of the fuel channels. This creates a zone of fuel-rich gas that is later convected into the combustion chamber when the velocity in the nozzle increases. The associated convective delay leads to a time-lag between oscillations of velocity and equivalence ratio that in turn causes a corresponding delay of the heat release response of the flame.

\section{Acknowledgments}

The work was funded by the German Aerospace Center (DLR) within the project IVTAS. The authors gratefully acknowledge W. Meier from DLR Institute of Combustion Technology for fruitful discussions about the PRECCINSTA burner and his support of this work, and F. Grimm for valuable help with the data analysis.

\section{References}

[1] A. H. Lefebvre, D. R. Ballal, Gas turbine combustion: alternative fuels and emissions, CRC Press, Boca Raton, USA, 2010.

[2] T. Lieuwen, V. Yang (Eds.), Combustion instabilities in gas turbine engines, Progress in Astronautics and Aeronautics, Vol. 210, AIAA, Washington, DC, USA, 2005.

[3] H. Pitsch, Large-Eddy Simulation of Turbulent Combustion, Annu. Rev. Fluid Mech. 38 (2006) 453-482.

[4] L. Y. Gicquel, G. Staffelbach, T. Poinsot, Large Eddy Simulations of gaseous flames in gas turbine combustion chambers, Prog. Energy Combust. Sci. 38 (2012) 782-817.

[5] G. Boudier, N. Lamarque, G. Staffelbach, L. Gicquel, T. Poinsot, Thermoacoustic stability of a helicopter gas turbine combustor using large-eddy simulations, Int. J. Aeroacoustics 8 (2009) 69-94.

[6] B. Franzelli, E. Riber, L. Gicquel, T. Poinsot, Large Eddy Simulation of combustion instabilities in a lean partially premixed swirled flame, Combust. Flame 159 (2012) 621-637.

[7] H.-G. Li, P. Khare, H.-G. Sung, V. Yang, A Large-Eddy-Simulation Study of Combustion Dynamics of Bluff-Body Stabilized Flames, Combust. Sci. Technol. 188 (2016) 924-952.

[8] S. Hermeth, G. Staffelbach, L. Gicquel, T. Poinsot, LES evaluation of the effects of equivalence ratio fluctuations on the dynamic flame response in a real gas turbine combustion chamber, Proc. Combust. Inst. 34 (2013) 3165-3173.

[9] I. Hernandez, G. Staffelbach, T. Poinsot, J. C. Romn Casado, J. B. W. Kok, LES and acoustic analysis of thermo-acoustic instabilities in a partially premixed model combustor, C. R. Mecanique 341 (2013) 121-130.

[10] H. Krediet, C. Beck, W. Krebs, J. Kok, Saturation mechanism of the heat release response of a premixed swirl flame using LES, Proc. Combust. Inst. 34 (2013) 1223-1230.

[11] G. Lartigue, U. Meier, C. Brat, Experimental and numerical investigation of self-excited combustion oscillations in a scaled gas turbine combustor, Appl. Therm. Eng. 24 (2004) 1583-1592.

[12] W. Meier, P. Weigand, X. Duan, R. Giezendanner-Thoben, Detailed characterization of the dynamics of thermoacoustic pulsations in a lean premixed swirl flame, Combust. Flame 150 (2007) 2-26.

[13] T. Lieuwen, B. T. Zinn, The role of equivalence ratio oscillations in driving combustion instabilities in low NOx gas turbines, Symp. (Int.) Combust. 27 (1998) 1809-1816.

[14] B. Schuermans, F. Guethe, D. Pennel, D. Guyot, C. O. Paschereit, Thermoacoustic modeling of a gas turbine using transfer functions measured under full engine pressure, J. Eng. Gas Turbines Power 132 (2010) 111503.

[15] B. Čosič, S. Terhaar, J. P. Moeck, C. O. Paschereit, Response of a swirlstabilized flame to simultaneous perturbations in equivalence ratio and velocity at high oscillation amplitudes, Combust. Flame 162 (2015) 10461062 .
[16] M. Stöhr, Z. Yin, W. Meier, Interaction between velocity fluctuations and equivalence ratio fluctuations during thermoacoustic oscillations in a partially premixed swirl combustor, Proc. Combust. Inst. 36 (2016), doi: 10.1016/j.proci.2016.06.084 .

[17] S. Roux, G. Lartigue, T. Poinsot, U. Meier, C. Brat, Studies of mean and unsteady flow in a swirled combustor using experiments, acoustic analysis, and large eddy simulations, Combust. Flame 141 (2005) 40-54.

[18] V. Moureau, P. Minot, H. Pitsch, C. Brat, A ghost-fluid method for large-eddy simulations of premixed combustion in complex geometries, J. Comput. Phys. 221 (2007) 600-614.

[19] B. Fiorina, R. Vicquelin, P. Auzillon, N. Darabiha, O. Gicquel, D. Veynante, A filtered tabulated chemistry model for LES of premixed combustion, Combust. Flame 157 (2010) 465-475.

[20] V. Moureau, P. Domingo, L. Vervisch, From Large-Eddy Simulation to Direct Numerical Simulation of a lean premixed swirl flame: Filtered laminar flame-PDF modeling, Combust. Flame 158 (2011) 1340-1357.

[21] J.-M. Lourier, B. Noll, M. Aigner, Large Eddy Simulation of a Thermoacoustic Instability within a Swirl-Stabilized Burner using Impedance Boundary Conditions, ASME Turbo Expo (2014) GT2014-26200.

[22] P. Wang, N. Platova, J. Fröhlich, U. Maas, Large Eddy Simulation of the PRECCINSTA burner, Int. J. Heat Mass Transfer 70 (2014) 486-495.

[23] T. Schönfeld, T. Poinsot, Influence of boundary conditions in LES of premixed combustion instabilities, CTR Annual Research Briefs (1999) 7384.

[24] W. Polifke, C. Wall, P. Moin, Partially reflecting and non-reflecting boundary conditions for simulation of compressible viscous flow, J. Comput. Phys. 213 (2006) 437-449.

[25] P. A. Hield, M. J. Brear, Comparison of open and choked premixed combustor exits during thermoacoustic limit cycle, AIAA J. 46 (2008) 517526.

[26] A. Huber, P. Romann, W. Polifke, Filter-Based Time-Domain Impedance Boundary Conditions for CFD Applications, ASME Turbo Expo (2008) GT2008-51195.

[27] A. Widenhorn, B. Noll, M. Aigner, Impedance Boundary Conditions for the Numerical Simulation of Gas Turbine Combustion Systems, ASME Turbo Expo (2008) GT2008-50445.

[28] R. Kaess, A. Huber, W. Polifke, A Time-Domain Impedance Boundary Condition for Compressible Turbulent Flow, 14th AIAA/CEAS Aeroacoustics Conference (2008) AIAA 2008-2921.

[29] G. Kelsall, C. Troger, Prediction and control of combustion instabilities in industrial gas turbines, Appl. Therm. Eng. 24 (2004) 1571-1582.

[30] J.-M. Lourier, A. Widenhorn, B. Noll, M. Stöhr, M. Aigner, Numerical Analysis of the Acoustic Transfer Behaviour of Pressure Ducts Utilised for Microphone Measurements in Combustion Chambers, ASME Turbo Expo (2010) GT2010-22805.

[31] D. Veynante, L. Vervisch, Turbulent combustion modeling, Prog. Energy Combust. Sci. 28 (2002) 193-266.

[32] M. Di Domenico, Numerical Simulation of Soot Formation in Turbulent Flows, Ph.D. thesis, University of Stuttgart, 2008.

[33] J. Ferziger, P. Milovan, Computational Methods for Fluid Dynamics, New York: Springer, 2002.

[34] A. Chorin, Numerical solution of the Navier-Stokes equations, Math. Comput. 22 (1968) 745-762.

[35] V. Moureau, C. Bérat, H. Pitsch, An efficient semi-implicit compressible solver for large-eddy simulations, J. Comput. Phys. 226 (2007) 12561270 .

[36] J.-M. Lourier, M. Di Domenico, B. Noll, M. Aigner, Implementation of an Efficient Pressure-Based CFD Solver for Accurate Thermoacoustic Computations, 18th AIAA/CEAS Aeroacoustics Conferences (2012) AIAA 2012-2089.

[37] J.-M. Lourier, B. Noll, M. Aigner, Extension of a Compressible PressureBased Solver for Reacting Flows, 19th AIAA/CEAS Aeroacoustics Conferences (2013) AIAA 2013-2101.

[38] P. Gerlinger, Investigation of an assumed PDF approach for finite-rate chemistry, Combust. Sci. Technol. 175 (2003) 841-872.

[39] J. M. Boyde, P. Le Clercq, M. Di Domenico, M. Aigner, Extension of the turbulent flame speed closure model to ignition in multiphase flows, Combust. Flame 160 (2013) 351-365.

[40] A. Fiolitakis, P. Ess, P. Gerlinger, M. Aigner, Modeling of heat transfer and differential diffusion in transported PDF methods, Combust. Flame 161 (2014) 2107-2119. 
[41] B. Magnussen, On the structure of turbulence and a generalized eddy dissipation concept for chemical reaction in turbulent flow, 19th AIAA Aerospace Science Meeting (1981) AIAA 1981-42.

[42] M. Di Domenico, P. Gerlinger, M. Aigner, Development and validation of a new soot formation model for gas turbine combustor simulations, Combust. Flame 157 (2010) 246-258.

[43] T. Blacha, M. Di Domenico, P. Gerlinger, M. Aigner, Soot predictions in premixed and non-premixed laminar flames using a sectional approach for PAHs and soot, Combust. Flame 159 (2012) 181-193.

[44] M. Köhler, K. P. Geigle, T. Blacha, P. Gerlinger, W. Meier, Experimental characterization and numerical simulation of a sooting lifted turbulent jet diffusion flame, Combust. Flame 159 (2012) 2620-2635.

[45] G. Eckel, M. Rachner, P. Le Clercq, M. Aigner, Semi-Empirical Model for the Unsteady Shear Breakup of Liquid Jets in Cross-Flow, Atomization Spray. 26 (2016) 687-712.

[46] S. Föller, W. Polifke, Determination of Acoustic Transfer Matrices via Large Eddy Simulation and System Identification, 16th AIAA/CEAS Aeroacoustics Conference (2010) AIAA 2010-3998.

[47] B. Gunasekaran, J. J. McGuirk, Mildly-compressible pressure-based CFD methodology for acoustic propagation and absorption prediction, ASME Turbo Expo (2011) GT2011-45316.

[48] F. R. Menter, Review of the shear-stress transport turbulence model experience from an industrial perspective, Int. J. Comput. Fluid Dyn. 23 (2009) 305-316.

[49] T. Poinsot, D. Veynante, Theoretical and numerical combustion, RT Edwards, Inc., 2005.

[50] F. Menter, Y. Egorov, The scale-adaptive simulation method for unsteady turbulent flow predictions. Part 1: theory and model description, Flow Turbul. Combust. 85 (2010) 113-138.

[51] L. Davidson, Large eddy simulations: how to evaluate resolution, Int. J. Heat Fluid Flow 30 (2009) 1016-1025.

[52] B. Magnussen, Modeling of NOx and soot formation by the Eddy Dissipation Concept, Int. Flame Research Foundation, 1st Topic Oriented Technical Meeting (1989) .

[53] S. Candel, D. Thevenin, N. Darabiha, D. Veynante, Progress in numerical combustion, Combust. Sci. Technol. 149 (1999) 297-337.

[54] K. Kuo, Principles of Combustion, Wiley, Hoboken, USA, 2nd edn., 2005.

[55] A. Kazakov, M. Frenklach, Reduced Reaction Sets based on GRIMech 1.2, http://www.me.berkeley.edu/drm/, University of California, Berkeley, URL http://www.me.berkeley.edu/drm/, last checked 10.02.2014

[56] G. Smith, D. Golden, M. Frenklach, N. Moriarty, B. Eiteneer, M. Goldenberg, T. Bowman, R. Hanson, S. Song, W. Gardiner Jr., V. Lissianski, Z. Qin, GRI-Mech, http://www.me.berkeley.edu/gri_mech/, URL http://www.me.berkeley.edu/gri_mech/, last checked 10.02.2014.

[57] S. Girimaji, Assumed $\beta$-pdf model for turbulent mixing: Validation and extension to multiple scalar mixing, Combust. Sci. Technol. 78 (1991) $177-196$.

[58] F. Lockwood, A. Naguib, The prediction of the fluctuations in the properties of free, round-jet, turbulent, diffusion flames, Combust. Flame 24 (1975) 109-124.

[59] J.-M. Lourier, C. Eberle, B. Noll, M. Aigner, Influence of turbulencechemistry interaction modeling on the structure and the stability of a swirl-stabilized flame, ASME Turbo Expo (2015) GT2015-43174.

[60] Y. Özyörük, L. N. Long, A time-domain implementation of surface acoustic impedance condition with and without flow, 2nd AIAA and CEAS Aeroacoustics Conference (1996) AIAA 96-1663.

[61] K. Fung, H. Ju, Time-domain Impedance Boundary Conditions for Computational Acoustics and Aeroacoustics, Int. J. Comput. Fluid Dyn. 18 (2004) 503-511.

[62] J.-M. Lourier, A. Huber, B. Noll, M. Aigner, Numerical Analysis of Indirect Combustion Noise Generation Within a Subsonic Nozzle, AIAA J. 52 (2014) 2114-2126.

[63] R. Munt, Acoustic transmission properties of a jet pipe with subsonic jet flow: I. The cold jet reflection coefficient, J. Sound Vib. 142 (1990) 413436.

[64] A. da Silva, G. Scavone, A. Lefebvre, Sound reflection at the open end of axisymmetric ducts issuing a subsonic mean flow: A numerical study, J. Sound Vib. 327 (2009) 507-528
[65] R. Bilger, S. Stårner, R. Kee, On reduced mechanisms for methane-air combustion in nonpremixed flames, Combust. Flame 80 (1990) 135-149.

[66] M. Stöhr, C. Arndt, W. Meier, Effects of Damköhler number on vortexflame interaction in a gas turbine model combustor, Proc. Combust. Inst. 34 (2013) 3107-3115.

[67] K. Oberleithner, M. Stöhr, S. H. Im, C. M. Arndt, A. M. Steinberg, Formation and flame-induced suppression of the precessing vortex core in a swirl combustor: experiments and linear stability analysis, Combust. Flame 162 (2015) 3100-3114.

[68] Y. See, M. Ihme, Large eddy simulation of a partially-premixed gas turbine model combustor, Proc. Combust. Inst. 35 (2015) 1225-1234.

[69] K. Kim, J. Lee, B. Quay, D. Santavicca, Response of partially premixed flames to acoustic velocity and equivalence ratio perturbations, Combust. Flame 157 (2010) 1731-1744 